\title{
Qualitative Comparative Analysis (QCA) in der Soziologie - Perspektiven, Potentiale und Anwendungsbereiche Qualitative Comparative Analysis (QCA) and Sociology - Perspectives, Potential, and Areas of Application
}

\author{
Jonas Buche* \\ Goethe-Universität Frankfurt, Fachbereich Gesellschaftswissenschaften, Institut für Politikwissenschaft, \\ Theodor-W.-Adorno-Platz 6, 60629 Frankfurt am Main, Germany. \\ buche@soz.uni-frankfurt.de
}

\author{
Markus B. Siewert* \\ Goethe-Universität Frankfurt, Fachbereich Gesellschaftswissenschaften, Institut für Politikwissenschaft, \\ Theodor-W.-Adorno-Platz 6, 60629 Frankfurt am Main, Germany. \\ siewert@soz.uni-frankfurt.de
}

\begin{abstract}
Zusammenfassung: Qualitative Comparative Analysis (QCA) wurde von dem Soziologen Charles C. Ragin als Verbindung von fallorientierten, konfigurativen Ansätzen und mengentheoretischem Denken präsentiert. Mittlerweile hat sich QCA - von Ragin und anderen weiterentwickelt - als mengentheoretischer Ansatz zur Untersuchung sozialer Phänomene im sozialwissenschaftlichen Methodenkanon etabliert. Der vorliegende Beitrag zielt darauf ab, Forschungsperspektiven und -potentiale von QCA als (relativ) junge Methode für soziologische Fragestellungen aufzuzeigen. Auf der Grundlage einer Rundschau von 77 publizierten, soziologischen Zeitschriftenartikeln wird einerseits ein breiter Überblick über Anwendungsbereiche, aktuelle Trends und Entwicklungen von QCA in der Soziologie gegeben. Andererseits werden am Beispiel der publizierten Studien die einzelnen Analyseschritte einer QCA besprochen und dabei gängige Fallstricke aufgezeigt, wobei sowohl practiced practices als auch best practices in ihrer Anwendung herausgearbeitet werden.
\end{abstract}

Schlagworte: Qualitative Comparative Analysis (QCA); fuzzy-set analysis; mengentheoretisches Denken; Evaluation von QCA-Anwendungen; Standards „guter“ Praxis.

\begin{abstract}
Summary: The sociologist Charles C. Ragin originally introduced Qualitative Comparative Analysis (QCA), which combines ideas of case-oriented research, configurational thinking, and set-theoretical logic. Since then - as further developed by Ragin and others - QCA has become an established tool within social science methodology which uses a set-theoretical approach to analyze social phenomena. This paper aims at highlighting perspectives and potentials which QCA as a (relatively) new method is able to offer to address sociological research questions. In order to do so, seventyseven publications in the field of sociology are reviewed. On this basis a broad overview is given regarding applications, trends, and developments of QCA, on the one hand. On the other, crucial steps within QCA are examined and potential pitfalls discussed by exemplifying practiced practices and best practices.
\end{abstract}

Keywords: Qualitative Comparative Analysis (QCA); Fuzzy-set Analysis; Set-theoretical Thinking; QCA-application Evaluation; Standards of "Good” Practice.

* Die Autoren danken den Teilnehmer*innen der AdHoc-Gruppe „Qualitative Comparative Analysis (QCA). Perspektiven für die soziologische Forschung " auf dem 37. Kongress der DGS in Trier für die hilfreiche Diskussion. Ein besonderer Dank gebührt Daniela Grunow, Carsten G. Ullrich, Antje Buche und Claudius Wagemann sowie zwei anonymen Gutachter*innen und dem Herausgeberkreis der ZfS für zahlreiche wertvolle und anregende Kommentare. Zudem bedanken wir uns bei Yulia Aleshchenkova und Christoph Klement für die Zuarbeit.

\section{Einleitung}

Die Publikation „The Comparative Method“ (1987) des amerikanischen Soziologen Charles C. Ragin ist in vielerlei Hinsicht bahnbrechend; in erster Linie jedoch legte sie den Grundstein für eine neue methodologische Perspektive in den Sozialwissenschaften: der Verbindung von fallorientierten, konfigurativen Ansätzen und mengentheoretischem Denken. Qualitative Comparative Analysis (QCA) 
ist hierfür gleichsam zum Synonym geworden. In den darauffolgenden Jahren von Ragin (2000, 2008) und anderen (siehe u.a. Rihoux \& Ragin 2009; Schneider \& Wagemann 2012) weiterentwickelt, hat sich QCA als mengentheoretischer Ansatz zur Untersuchung sozialer Phänomene zunehmend im sozialwissenschaftlichen Methodenkanon etabliert. ${ }^{1}$

Insbesondere seit der Einführung sogenannter fuzzy sets - was etwas sperrig mit „unscharfen Mengen“ (Wagemann \& Schneider 2003) übersetzt werden kann - ist ein gewisser Boom um QCA zu beobachten, der sich neben Lehrbüchern und zahlreichen methodologischen Artikeln zuvorderst in der steigenden Zahl von QCA-Anwendungen ausdrückt (vgl. Rihoux et al. 2013). Zieht man etwa Publikationen in Zeitschriften mit Begutachtungsverfahren als Indikator für die Popularität von QCA heran, so wurden zwischen 1987 und 2011 die zweitmeisten aller QCA-Studien zu soziologischen Themen veröffentlicht. ${ }^{2}$ Wirft man zudem einen Blick auf die jährlichen Publikationsraten rangiert die Soziologie gemeinsam mit Politikwissenschaften und Business und Management stets unter den ersten drei Anwendungsfeldern (Rihoux et al. 2013: 177).

An der deutschsprachigen Soziologie scheint die „Ragin Revolution“ (Vaisey 2009) allerdings weitgehend vorübergegangen zu sein. So sind bis dato lediglich zwei soziologische Studien in deutscher Sprache in Zeitschriften mit Begutachtungsverfahren erschienen, die auf einer QCA beruhen (Hörisch 2012; Laux 2015). Diese Tatsache bildet den Ausgangspunkt des vorliegenden Beitrags, der drei verschiedene Zielsetzungen verfolgt: Erstens wird eine knappe Einführung zum Grundverständnis von QCA gegeben, welche die originäre Perspektive auf Mengentheorie beruhender Ansätze erläutert. Zweitens wird auf der Grundlage einer Rundschau von 77 Artikeln aus soziologischen Journals ein breiter Überblick über die Anwendung von QCA in der Soziologie gegeben. Drittens werden am Beispiel zahlreicher publizierter Studien die einzelnen Analyseschritte einer QCA besprochen und dabei diverse Tücken, aber auch best practices in ihrer Anwendung herausgearbeitet. In der Zusammenschau zielt der vorliegende Beitrag darauf ab, For-

\footnotetext{
${ }^{1}$ Für einen sehr guten historischen Überblick über die Entwicklung von QCA in den letzten 25 Jahren siehe Marx et al. 2014.

2 Der Anteil der Soziologie in diesem Zeitraum lag bei etwa $34 \%$ (gemeinsam mit anthropologischen Fragestellungen). Die meisten Anwendungen finden sich mit $51 \%$ im Bereich der Politikwissenschaft. Siehe Rihoux et al. 2013: 177.
}

schungspotentiale von QCA als (relativ) junger Methode für soziologische Fragestellungen aufzuzeigen, aktuelle Trends und Entwicklungen soziologischer QCA-Anwendungen zu präsentieren und grundlegende forschungspraktische Probleme und Fallstricke am Beispiel publizierter QCA-Studien aus der Soziologie zu diskutieren.

Hierzu werden in einem ersten Schritt zentrale Elemente von QCA als einem mengentheoretischen Forschungsansatz vorgestellt und erläutert. Im dritten Abschnitt wird eine Rundschau soziologischer QCA-Studien vorgelegt, welche Fragen und Analyseebenen erläutern. Im vierten Abschnitt wird die Vorgehensweise bei einer QCA skizziert, wobei anhand publizierter Studien Probleme und Fallstricke in der Analyse aufgezeigt und diskutiert werden. In Abschnitt fünf werden schließlich drei QCA-Anwendungen - je eine auf Makro-, Meso- und Mikroebene - im Sinne von best practices präsentiert. Abschließend wird argumentiert, dass mengentheoretische Methoden wie QCA einen deutlichen Mehrwert gerade für soziologische Fragestellungen aufweisen und den soziologischen Methodenkanon sinnvoll zu ergänzen vermögen.

\section{Was es heißt, soziale Phänomene mit QCA zu betrachten}

Charles Ragin präsentierte QCA ursprünglich als die vergleichende Methode für Forschungsdesigns mit geringer bis mittlerer Fallzahl (Ragin 1987, 2000). Auch wenn bis heute zahlreiche Studien die Anwendung einer QCA mit dem Argument der mittleren Fallzahl rechtfertigen, so ist dieses Kriterium keineswegs das entscheidende. Wesentlich zentraler ist vielmehr, dass mit QCA eine mengentheoretische Forschungsperspektive eingenommen wird (Schneider \& Wagemann 2012: 8-12). Was dies im Einzelnen bedeutet, wird in den folgenden Abschnitten erläutert.

So steht die Suche nach notwendigen und hinreichenden Bedingungen im Zentrum einer jeden QCA (vgl. Ragin 2008: 29-68; Schneider \& Wagemann 2012: 56-90). Nehmen wir als hypothetisches Beispiel einmal an, wir sind an Begründungen für eine ,hohe Lebenszufriedenheit' interessiert, wobei ein möglicher Erklärungsfaktor hierfür eine ,hervorragende Gesundheit' sein könnte. Um nun zu prüfen, ob ein sehr guter Gesundheitszustand (X) eine notwendige Bedingung für eine hohe Lebenszufriedenheit (Y) darstellt, muss immer dann, wenn $\mathrm{Y}$ vorliegt, auch $\mathrm{X}$ gegeben sein. Eine hohe Lebenszufriedenheit könnte in diesem Fall nicht 
Abbildung 1a:

Abbildung 2a:

$2 \times 2$ Tabelle für notwendige Bedingungen

$2 \times 2$ Tabelle für hinreichende Bedingungen

\begin{tabular}{|c|c|c|c|c|c|}
\hline 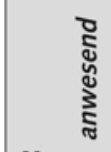 & Fälle nicht erlaubt & Fälle erlaubt & 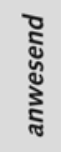 & $\begin{array}{l}\text { Fälle erlaubt; } \\
\text { aber irrelevant }\end{array}$ & Fälle erlaubt \\
\hline $\begin{array}{l}\text { క్ } \\
\text { ญे } \\
\text { స్ }\end{array}$ & $\begin{array}{l}\text { Fälle erlaubt; } \\
\text { aber irrelevant }\end{array}$ & $\begin{array}{l}\text { Fälle erlaubt; } \\
\text { aber irrelevant }\end{array}$ & 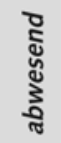 & $\begin{array}{l}\text { Fälle erlaubt; } \\
\text { aber irrelevant }\end{array}$ & Fälle nicht erlaubt \\
\hline & abwesend & anwesend & & abwesend & anwesend \\
\hline
\end{tabular}

Abbildung 1b:

Venn-Diagramm für notwendige Bedingungen

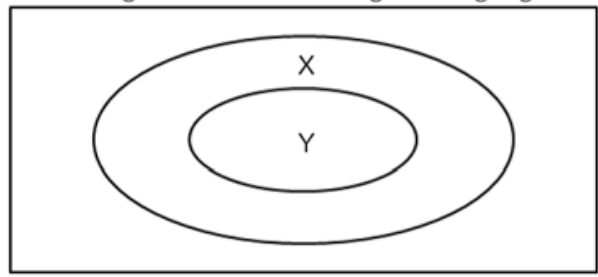

Abbildung 1c:

XY-Plot für notwendige Bedingungen

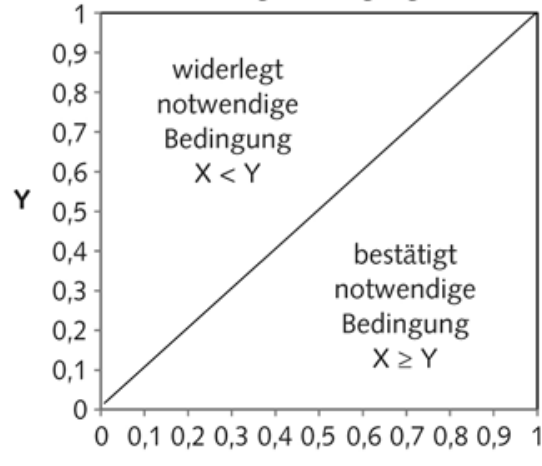

$\mathrm{x}$
Abbildung $2 b$ :

Venn-Diagramm für hinreichende Bedingungen

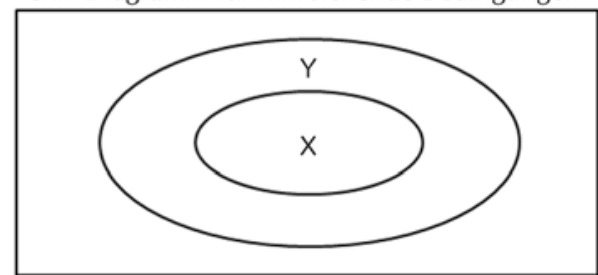

Abbildung 2c:

XY-Plot für hinreichende Bedingungen

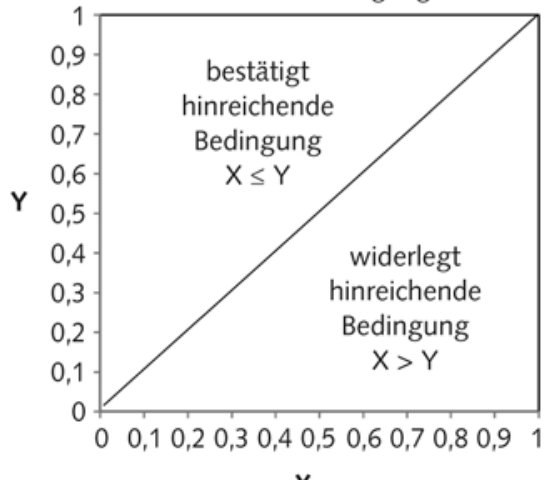

Abbildungen 1 und 2: Notwendige und hinreichende Bedingungen

ohne eine hervorragende Gesundheit auftreten. Aus mengentheoretischer Perspektive ließe sich diese Beziehung so beschreiben, dass das Outcome eine Teilmenge (subset) der Bedingungsmenge bzw. die Bedingung eine Übermenge (superset) der Outcomemenge ist (siehe Abb.1a-1c). Eine hinreichende Bedingung hingegen verhält sich quasi spiegelbildlich zu einer notwendigen. So könnte eine ,hervorragende Gesundheit' als hinreichend bezeichnet werden für den Fall, dass wann immer jemand sehr gesund ist, auch die Lebenszufriedenheit hoch ist. Es dürften also keine Personen existieren, die Mitglied in der Menge, hervorragende Gesundheit ${ }^{\star}$ sind, zugleich aber Nicht-Mitglied in der Menge ,hohe Lebenszufriedenheit'. ${ }^{3}$ Eine Bedingung ist also dann hinreichend, wenn wann immer $\mathrm{X}$ auftritt, auch Y vorliegt; oder mengentheoretisch ausgedrückt: Die Menge aller sehr gesunden Personen ist eine Teilmenge (subset) aller Menschen mit hoher Lebenszufriedenheit (siehe Abb. 2a-c).

\footnotetext{
${ }^{3}$ Dies gilt natürlich nur insofern, als dass bei allen Personen die gleichen Hintergrundfaktoren (scope conditions) gegeben sind. Hier spielt die Fallauswahl hinsichtlich der Untersuchungsfrage eine zentrale Rolle für die kausale Homogenität und Generalisierbarkeit über die Fälle hinweg (siehe etwa Rohlfing 2012: 46f.).
} 
Mit QCA als mengentheoretischem Ansatz geht somit eine ganz spezifische Sichtweise auf soziale Phänomene einher. Ragin (1987: 16-17; 34-68, 2004) spricht hier auch von einer fallorientierten (caseoriented) Perspektive, die sich grundlegend von variablen-orientierten Ansätzen wie etwa statistischen Verfahren unterscheidet. Der Unterschied liegt bereits in den zentralen Untersuchungseinheiten, nämlich Mengen (sets), begründet. Mengen können als "zones of inclusion and exclusion" definiert werden, in welche Fälle „according to their fit within the boundaries of a set" zugeordnet werden (Mahoney 2010, zit. nach Schneider \& Wagemann 2012: 24; vgl. auch Verkuilen 2005). Die zu untersuchenden Fälle werden demnach als Konfigurationen unterschiedlicher Mengen verstanden, in denen Fälle einen bestimmten Grad an Mitgliedschaft aufweisen können. Beispielsweise kann ein Individuum volles Mitglied, volles Nicht-Mitglied oder partielles Mitglied - mehr innerhalb oder mehr außerhalb einer Menge, ggf. mit verschiedenen Abstufungen - in der Menge ,Menschen mit hoher Lebenszufriedenheit' sein. Mengen zeichnen sich folglich dadurch aus, dass sie sowohl quantitative Abstufungen (differences in degree) partieller Mitgliedschaften als auch qualitative Unterschiede (differences in kind) zwischen Nicht-Mitglied und Mitglied in einer Menge erfassen können (Schneider \& Wagemann 2012: 24-31; Ragin 2008: 29-34). ${ }^{4}$

Bei der Zuweisung der Mengenmitgliedschaften (Kalibrierung) kommt das qualitative, fallorientierte Moment gleich mehrfach zum Tragen: So erfolgt die Kalibrierung idealiter in einem iterativen Prozess des „back and forth between theory and empirical evidence“" (Ragin 1987: 78) auf Grundlage theoretischen und fallspezifischen Wissens in Kombination mit empirischen Informationen des Datenkorpus wie z. B. Mittelwerten oder der Verteilung der Daten. Im Gegensatz zu Variablen, welche die Varianz eines Indikators eins zu eins abbilden, werden Mengen in Bezug auf das zugrundeliegende Konzept kalibriert. Nehmen wir beispielsweise das Konzept ,hoher sozio-ökonomischer Status', welches wir über den Indikator monatliches

\footnotetext{
${ }^{4}$ Bei sogenannten fuzzy sets kann die Mengenmitgliedschaft zwischen voller Mitgliedschaft (Mitgliedschaftswert 1) und voller Nicht-Mitgliedschaft (Mitgliedschaftswert 0 ) in beliebig vielen Stufen partieller Mitgliedschaft variieren (z. B. Mitgliedschaftswert 0.8 für eine teilweise Mitgliedschaft oder 0.4 für eine teilweise Nicht-Mitgliedschaft). Eine spezielle Variante sind dabei sogenannte crisp sets, die lediglich eine Unterscheidung zwischen Mitgliedschaft und Nicht-Mitgliedschaft bieten und somit dichotom sind.
}

Einkommen erheben. Für eine volle Mitgliedschaft könnte nun ein Wert von über $5.579 €$ (aktuelles W3-Einstiegsgehalt in Hessen) festgelegt werden; damit würde allen Personen mit einem höheren monatlichen Einkommen, egal ob $5.580 €$ oder $10.000 €$, eine volle Mitgliedschaft in der Menge von Personen mit hohem sozio-ökonomischen Status zugewiesen. Damit wird durch die Kalibrierung von Mengen Varianz in den Indikatoren trunkiert Ragin spricht hier von irrelevanter Varianz (2008: 74). Hierbei obliegt es der Forscherin, wie sie das Konzept in Abhängigkeit ihrer Theorie, Fallauswahl und empirischen Informationen definiert.

Mengentheoretische Ansätze wie QCA besitzen darüber hinaus ein spezifisches Verständnis von sozialer Realität (grundlegend Ragin 2008: 176-187; Schneider \& Wagemann 2012: 76-89; Rohlfing 2012: 40-60; Goertz \& Mahoney 2012: 16-74). So sind Mengenbeziehungen erstens durch Asymmetrie geprägt, welche sich in verschiedenen Kontexten zeigt. So werden etwa bei notwendigen und hinreichenden Bedingungen unterschiedliche Annahmen geprüft. Bei notwendigen Bedingungen kann Y nicht ohne X vorliegen. Hier interessieren uns folglich nur solche Fälle, die auch tatsächlich das Outcome aufweisen: Ob Menschen ohne hohe Lebenszufriedenheit gesund sind oder nicht, beeinflusst die Aussage über eine notwendige Bedingung dagegen in keiner Weise. Umgekehrt sind bei hinreichenden Bedingungen lediglich solche Fälle für den Wahrheitsgehalt der Aussage ausschlaggebend, die $\mathrm{X}$ aufweisen. Dies bedeutet, dass auf der einen Seite solche Fälle, die sowohl X als auch Y aufzeigen, eine hinreichende Aussage bestätigen. Auf der anderen Seite widersprechen aber all diejenigen Fälle einer hinreichenden Bedingung, in denen Bedingung $\mathrm{X}$ vorliegt, aber nicht das Outcome Y. Alle Fälle ohne Bedingung $X$ sind hingegen irrelevant für die Aussage, dass X hinreichend für Y ist (siehe auch Abb. 1a und 2a).

Mengentheoretische Annahmen sind demnach nicht-linear. Bezogen auf das Beispiel Gesundheit und Lebenszufriedenheit würde ein linearer Zusammenhang etwa nicht nur beschreiben, dass mehr Gesundheit zu mehr Lebenszufriedenheit führt, sondern zugleich auch behaupten, dass weniger Gesundheit zu weniger Lebenszufriedenheit führt. Aus einer mengentheoretischen Perspektive hingegen würde man formulieren, wenn eine hervorragende Gesundheit, dann eine hohe Lebenszufriedenheit. Damit wird lediglich eine Aussage über den $\mathrm{Zu}-$ stand gemacht, wenn eine hervorragende Gesundheit vorliegt, allerdings nichts ausgesagt, was in ihrer Abwesenheit geschieht. 
Ein weiterer Aspekt von Asymmetrie liegt in der Kalibrierung von Mengen begründet. Demnach muss der positive Pol eines Konzepts sich nicht zwangsläufig symmetrisch zum negativen Pol verhalten. Nehmen wir etwa wieder das Konzept ,sozio-ökonomischer Status'. Dieses Konzept kann einerseits symmetrisch verstanden werden, so dass das konzeptuelle Kontinuum beispielsweise zwischen ,arm' und ,reich' liegt. Es kann aber auch so konzeptuell erfasst werden, dass der negative Pol ,nicht-reich' ist, was eine grundsätzlich andere Kalibrierung erforderlich macht und auch in der Analyse zu unterschiedlichen Schlussfolgerungen führen kann. Dies wiederum sollte bei der Kalibrierung eines Konzepts als Menge beachtet werden (Goertz \& Mahoney 2012: 64-74, 161-165).

Zweitens ist die Perspektive einer QCA Y-zentriert, d.h. es wird - quasi in Rückschau - nach den Ursachen für ein bestimmtes Outcome (causes-of-effects) gesucht. Damit eng verbunden ist das konfigurative Verständnis, wonach Fälle als Konfigurationen von Bedingungen analysiert werden. Im Gegensatz zu X-zentrierten Forschungsdesigns zielt QCA somit nicht auf die Untersuchung durchschnittlicher Kausaleffekte (average causal effects) einzelner Erklärungsfaktoren; vielmehr wird nach Bedingungskombinationen gesucht, welche in ihrem Zusammenspiel ein bestimmtes Outcome erklären können. Zur Veranschaulichung können wir wieder auf das bekannte hypothetische Beispiel zurückgreifen: So können Individuen nicht nur (Nicht-)Mitglieder in der Menge ,gesunder Menschen' (A), sondern zugleich auch in der Menge ,Menschen in Partnerschaft ${ }^{\circ}$ (B) sein. Aus diesen zwei Bedingungen ergeben sich die vier Idealtypen ,gesunder Mensch in Partnerschaft ${ }^{6}\left(A^{*} B\right)^{5}$, , gesunder Mensch nicht in Partnerschaft" $\left(A^{*} \sim B\right)$ sowie ,nicht-gesunder Mensch in Partnerschaft ${ }^{\star}\left(\sim \mathrm{A}^{*} \mathrm{~B}\right)$ und ,nicht-gesunder Mensch nicht in Partnerschaft ${ }^{\circ}$ $(\sim A * \sim B)$. Erweitert um eine dritte Bedingung, etwa der Menge ,hoher sozio-ökonomischer Status‘ (C), ergäben sich bereits acht idealtypische Merkmalskombinationen.

In der Analyse von notwendigen und hinreichenden Bedingungen treten diese konfigurativen Elemente in Form von SUIN- und INUS-Bedingungen zu Tage. Das Akronym SUIN steht dabei für „sufficient but unnecessary part of a factor that is insufficient

\footnotetext{
${ }^{5}$ QCA basiert auf Boolescher Algebra. Das Multiplikationszeichen (*) steht für das logische UND, das Additionszeichen (+) für das logische ODER. Das Tildezeichen ( ) steht für die Abwesenheit der Menge. Ein Pfeil impliziert eine notwendige $(\leftarrow)$ oder hinreichende $(\rightarrow)$ Bedingung.
}

but necessary for the result" (Mahoney et al. 2009). Mit unserem obigen Beispiel könnte ein Ergebnis einer QCA etwa sein, dass die Kombination aus in einer Partnerschaft lebend ODER hohem sozio-ökonomischem Status eine notwendige Bedingung für eine hohe Lebenszufriedenheit darstellen. Der entsprechende Lösungsterm einer QCA sähe demnach wie folgt aus: $\mathrm{B}+\mathrm{C} \leftarrow \mathrm{Y}$. Die Bedingungen $\mathrm{B}$ und $\mathrm{C}$ wären in diesem Fall wechselseitig substituierend und damit SUIN-Bedingungen. Liegt beispielsweise keine Partnerschaft vor, muss der Single demnach einen hohen sozio-ökonomischem Status aufweisen, um überhaupt Lebenszufriedenheit erreichen $\mathrm{zu}$ können. INUS-Bedingungen sind hingegen solche, die ,insufficient but necessary part of a condition which is itself unnecessary but sufficient for the result" (Mackie 1974: 62) sind. Wiederum ausgehend von unserem Beispiel könnte eine Lösung einer QCA für eine hinreichende Bedingung sein: $A * B \rightarrow Y$. Dies würde bedeuten, dass eine hohe Lebenszufriedenheit durch die Kombination von gesunden, in einer Partnerschaft lebenden Menschen beschrieben werden kann. A UND B sind somit INUS-Bedingungen, da sie notwendiger Teil einer Bedingungskombination sind, die als solche hinreichend für das Outcome ist.

Drittens zielen Mengenbeziehungen darauf ab, äquifinale Erklärungspfade für ein Outcome aufzudecken. So könnte das obige Beispiel um einen weiteren Term erweitert werden und etwa $(\mathrm{A} * \mathrm{~B})+$ $\left(\sim \mathrm{B}^{*} \mathrm{C}\right) \rightarrow \mathrm{Y}$ lauten. Hier wären sowohl die Kombinationen aus hervorragender Gesundheit UND in einer Partnerschaft lebend $\left(\mathrm{A}^{*} \mathrm{~B}\right)$ als auch Single UND hoher sozio-ökonomischen Status $\left(\sim B^{*} C\right)$ hinreichende Bedingungen für das Outcome ,hohe Lebenszufriedenheit‘. Dabei zeigt sich wiederum die fallorientierte Perspektive von QCA, denn die äquifinalen Lösungsterme beschreiben oftmals unterschiedliche Fälle. Zudem verdeutlicht das Beispiel einen weiteren Aspekt der zuvor beschriebenen Asymmetrie, denn die Bedingung, in einer Partnerschaft lebend“ (B) ist in der ersten Konfiguration in ihrer Anwesenheit hinreichend für das Outcome, hingegen in der zweiten Kombination in ihrer Abwesenheit.

Die Entscheidung für ein Forschungsdesign mit einer QCA ist demnach eine Entscheidung für eine mengentheoretische Untersuchungsperspektive. Zusammenfassend bedeutet dies, dass erstens Konzepte als Mengen begriffen werden, in denen Fälle einen bestimmten Grad an Mitgliedschaft aufweisen können; dass zweitens ein Verständnis von komplexer sozialer Realität als konfigurativ, äquifinal und asymmetrisch vorliegt; und dass drittens 


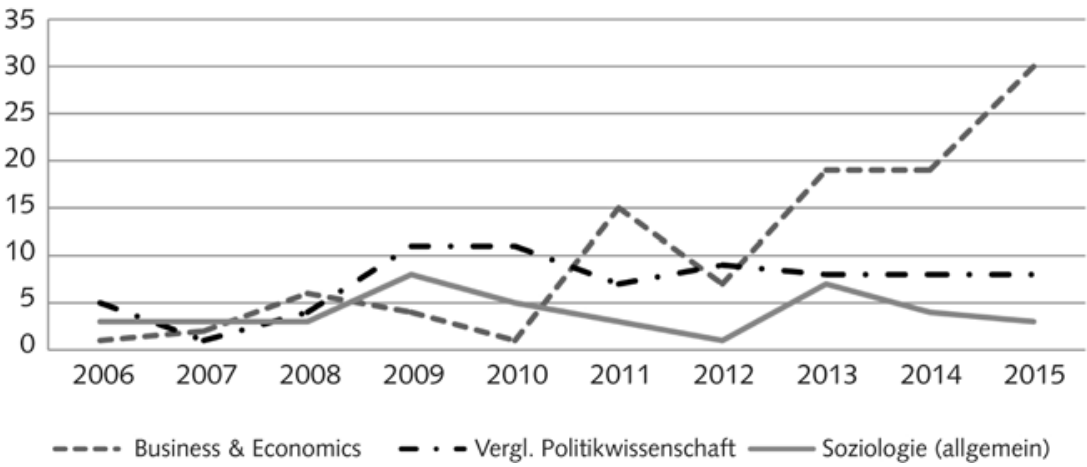

Abbildungen 3:

Anzahl publizierter Journalartikel nach Disziplin, 2006-2015

Quelle:

www.compasss.org,

Stand: 19. November 2015
Beziehungen zwischen Mengen als notwendige und hinreichende Bedingungen verstanden und untersucht werden können. Aufgrund dieser drei Merkmale eröffnen mengentheoretische Ansätze wie QCA also einen vollkommen anderen Blick auf soziale Phänomene als etwa korrelative Verfahren.

\section{QCA in der Soziologie: Eine Rundschau}

In der vergangenen Dekade hat sich QCA zweifelsohne als Forschungsdesign und -methode in den Sozialwissenschaften etabliert. Wirft man etwa einen Blick in die Datenbank publizierter Zeitschriftenartikel auf der Webseite www.compasss.org, so zeigt sich, dass allein im Jahr 2015 (Stand: 19. November 2015) bisher 91 Publikationen erschienen sind, die QCA als Methode einsetzen und sich der bisherige Trend der 2010er Jahre fortsetzt. So sind über die Hälfte aller in der Datenbank erfassten Publikationen, die seit „The Comparative Method“ (Ragin 1987) veröffentlicht wurden, in den letzten vier Jahren erschienen. Im Vergleich der Fachrichtungen fällt die Soziologie allerdings als Anwendungsgebiet von QCA leicht ab: Zwar rangiert sie gemeinsam mit der Politikwissenschaft und der Mikro-Ökonomie stets unter den ersten drei, doch ist gerade in den beiden anderen Disziplinen in jüngster Zeit ein größerer Zuwachs an Publikationen zu verzeichnen (siehe Abb. 3).

Der folgende Überblick basiert auf insgesamt 77 Studien, die über die COMPASSS-Datenbank erhoben wurden. ${ }^{6}$ Zwar handelt es sich hierbei um eine

\footnotetext{
${ }^{6}$ Bei der Fallauswahl beschränken wir uns auf Publikationen, die seit 2006 erschienen sind. In diesem Jahr wurden die Parameter Konsistenz und Abdeckung eingeführt (Ragin 2006), die eine wesentliche Neuerung für QCA darstellen. Neben den 40 Studien in der Rubrik, Soziologie (allgemein) ${ }^{`}$ wurden 37 weitere Artikel mittels der Daten-
}

nicht-repräsentative Auswahl, die nichtsdestotrotz ermöglicht, zentrale Trends zu QCA innerhalb der Soziologie herauszuarbeiten und zu illustrieren.

Aus der Rundschau der Zeitschriftenbeiträge ist zunächst die breite Spanne an soziologischen Fragestellungen und Gegenstandsbereichen herauszulesen, die mit Hilfe von QCA untersucht wird. Diese zeigt sich, erstens, anhand der verschiedenen Forschungsobjekte, welcher sich die untersuchten Studien annehmen. So behandelt knapp die Hälfte der Publikationen (35 von 77 Studien) makro-soziologische Themen zu Staaten (Laux 2015; Schneider \& Makszin 2014a; Svevo-Cianci et al. 2010) und subnationalen (Epple et al. 2014) oder lokalen Einheiten (Blackman 2013; Wollebæk, 2010; McVeigh et al. 2006). Darüber hinaus basiert rund ein Viertel der Studien (21 von 77) auf Daten, die auf einer mittleren Analyseebene angesiedelt sind; diese haben beispielsweise soziale Bewegungen (Giugni \& Nai 2013; Wright \& Schaffer Boudet 2012; Kröger 2011; Giugni \& Yamasaki 2009), Organisationen (Hodson et al. 2006; Grant et al. 2010) oder andere Gruppen (Vaisey 2007; Moritz et al. 2011) zum Gegenstand der Untersuchung. Ebenso viele Studien (21 von 77) betrachten schließlich die Mikro-Ebene individueller Akteure wie Schüler und Studierende (Glaesser \& Cooper 2011, 2012a, 2012b; Roberts et al. 2010; Shanahan et al. 2007, 2008), Beamte und Angestellte (Baltzer et al. 2011; Braun 2013) oder Basketball-Trainer (Savage \& Seebruck 2015).

Eng mit den Analysenebenen zusammenhängend, drückt sich die Vielfalt der Themen, zweitens, in den Daten aus, auf denen die einzelnen QCAs beru-

bank www.compasss.org in den Überblick aufgenommen, die ebenfalls soziologische Fragestellungen behandeln. Eine Übersicht findet sich im Online-Appendix. Stand der erfassten Zeitschriftenbeiträge ist der 19. November 2015 . 
hen. Die allermeisten Studien greifen auf aggregierte Indikatoren oder Indizes (McLevey 2014; Hafner-Fink et al. 2013; Grant et al. 2010; Ishida et al. 2006) zurück und oftmals werden auch Daten aus Surveys (Glaesser \& Cooper 2011, 2012a, 2012b; Longest \& Thoits 2012; Vaisey 2007) oder Interviews (Giugni \& Nai 2013; Marr 2012; Baltzer et al. 2011) zur Kalibrierung der Mengen herangezogen. Darüber hinaus finden sich aber auch qualitative Methoden der Datenerhebungen: So nutzen einige Anwendungen Informationen aus qualitativen Inhaltsanalysen (Laux 2015; Amenta et al. 2009), ethnographische Studien (Moritz et al. 2011; Hodson et al. 2006) oder teilnehmenden Beobachtungen (Vaisey 2007; Britt \& Evans 2007) als Grundlage ihrer Untersuchungen.

Drittens zeigt sich die Bandbreite soziologischer Forschung, die sich QCA bedient, in den Forschungsfragen und -themen der publizierten Anwendungen. Von der Resilienz einzelner Betroffener von Naturkatastrophen (Perry \& Schafer 2014) zur Entwicklung von Wohlfahrtsstaatsregimen (Ahn \& Seung-yoon Lee 2012; Jang 2009), vom Ausmaß der Risiken bei der Internetnutzung durch Kinder (Bauwens et al. 2009) zu Ungleichheiten am Arbeitsmarkt (Bentele 2013; Crowley 2012, 2013, 2014; Da Roit \& Weicht 2013), von sozialen Netzwerken älterer Personen (Haynes et al. 2010, 2013; Haynes 2011) zu gesundheitssoziologischen Ungleichheitsfragen (Blackman et al. 2011; Blackman \& Dunstan 2010, Blackman 2008) - stets wird in vergleichender Perspektive nach dem Zusammenspiel von Bedingungen gefragt, unter denen ein bestimmtes Outcome auftritt oder nicht auftritt.

Mit Blick auf die unterschiedlichen Varianten von QCA ist bemerkenswert, dass in der Soziologie bis heute häufiger die auf die dichotome Unterscheidung zwischen Mitgliedschaft und Nicht-Mitgliedschaft begrenzte crisp set QCA angewendet wird als fuzzy sets (in 43 von 77 Studien; siehe hierzu auch Rihoux et al. 2013: 176). Dies ist insofern erstaunlich, als dass sich soziale Phänomene in der Regel nicht (eindeutig) dichotomisieren lassen und die Entwicklung von fuzzy sets eine Antwort hierauf darstellt.

In Bezug auf die Anzahl der einbezogenen Erklärungsfaktoren kann konstatiert werden, dass drei Viertel der Studien (58 von 77) auf einer mittleren Zahl an Bedingungen - d.h. zwischen drei und sechs - beruhen. Allerdings werden in ca. jeder zehnten Studie (8 von 77) neun (Harris-White et al. 2013; Hussain \& Howard 2013; Melinder 2007) oder gar bis zu elf (Eng \& Woodside 2012; McAdam et al. 2010) Bedingungen in die Untersuchung eingespeist. Eine einzige Studie beruht hingegen nur auf dem Zusammenspiel zweier Erklärungsfaktoren (Stevenson 2013).

Die Anzahl der Bedingungen muss allerdings - wie später noch erläutert wird - immer auch im Verhältnis zur Fallzahl betrachtet werden. Hier zeigt sich, dass QCA in der Tat als Methode zur Analyse mittlerer Fallzahlen angewendet wird, denn eine große Mehrheit der Studien (48 von 77) beruht auf zehn bis ca. 50 Fällen. Allerdings ist dies nicht ausschließlich so: Während gerade einmal fünf Prozent der Studien (4 von 77) weniger als zehn Fälle untersuchen (Bleijenbergh \& Roggeband 2007; Ignatow 2011; Achilov \& Shaykhutdinov 2013; Da Roit \& Weicht 2013), finden sich zahlreiche Publikationen, die mehrere hundert (Perry \& Schafer 2014; Longest \& Thoits 2012; Grant et al. 2009a, 2009b; Hodson et al. 2006) oder gar tausende Fälle (Amenta et al. 2009; Shanahan et al. 2007, 2008; Glaesser \& Cooper 2011, 2012a, 2012b) einer QCA unterziehen.

\section{Beispielstudien: Analyseschritte und potentielle Fallstricke einer QCA}

Wie in Abschnitt 2 gezeigt wurde, ist QCA als Forschungsansatz tief in der qualitativ-empirischen, fallorientierten Wissenschaftstradition verwurzelt (Ragin 1994; Goertz \& Mahoney 2012; Blatter \& Haverland 2012). Auch wenn QCA als Methode auf Boolescher Algebra basiert und diverse Formeln, Algorithmen und Softwares zum Einsatz kommen, so muss die Forscherin in jeder Analysephase eine Vielzahl (qualitativer) Entscheidungen treffen, die sich aus ihrem Theoriewissen sowie ihrer Fallexpertise speisen sollten. So wird im Folgenden zwar eine Art „rezeptartiger" Ablauf einer QCA skizziert (Wagemann 2015: 436), was allerdings nicht zu einer rein mechanischen Anwendung weder der Software noch existierender Maßzahlen führen darf. Vielmehr sollte jede QCA-Anwendung zwar minimale Standards beachten (vgl. Schneider \& Wagemann 2010, 2012: 275-304); die zahlreichen Entscheidungen sollten aber transparent dargestellt werden (siehe Wagemann \& Schneider 2015) und stets an eine fallorientierte Perspektive rückgebunden werden.

\section{Schritt 1 - Kalibrierung von Mengen}

Empirische Daten, die in eine QCA einfließen sollen, müssen in einem ersten Schritt zu Mengen geformt werden. Dieser Schritt wird Kalibrierung ge- 
nannt. Diese Phase beinhaltet bereits ein starkes qualitatives Element, da die einer QCA zugrundeliegenden Konzepte in einem iterativen Prozess zwischen theoretischem Wissen und Empirie entwickelt, spezifiziert und zu Mengen kalibriert werden. Während die spätere Analyse mit Hilfe geeigneter Software in der Regel eher weniger Zeit in Anspruch nimmt, sind es die, Vorarbeiten' wie Konzeptformierung und Kalibrierung der Mengen, die den Großteil der analytischen Arbeit ausmachen.

Um Mengen sinnvoll kalibrieren zu können, d.h. den untersuchten Fällen begründet Mengenmitgliedschaften zuweisen zu können, bedarf es einer klaren Konzeptformierung (siehe etwa Goertz 2006; Adcock \& Collier 2001). Dies lässt sich in einem ersten Schritt dadurch erreichen, dass Mengen mit einem aussagekräftigen Label versehen werden, welches Mitgliedschaftsaussagen möglich macht. Während zum Beispiel ein Mitgliedschaftswert von 0.8 in Mengen wie ,Mobilisierung' (Wright \& Schaffer Boudet 2012: 743), ,Teilzeitarbeit' (Lee 2013: 338) oder ,Sozialbeziehung' (Marr 2012: 991) wenig aussagekräftig ist, sähe dies für alternativ bezeichnete Mengen wie ,Soziale Bewegungen mit hohem Mobilisierungsgrad', ,Staaten mit hohem Anteil an Teilzeitarbeit' oder,Personen mit vielen Sozialbeziehungen' bereits anders aus. Adjektive bieten sich als Labels auch deshalb an, weil sie asymmetrische Konzepte zu beschreiben vermögen. So verbleibt beispielsweise die Mengenmitgliedschaft einer Person mit einer vergleichsweise durchschnittlichen Anzahl von Sozialbeziehungen in der Menge ,Sozialbeziehungen' unklar; sie kann sowohl Mitglied als auch Nicht-Mitglied sein. In der alternativen Menge ,Personen mit vielen Sozialbeziehungen' wird dieser Fall kein Mitglied sein, da er eben nicht viele, sondern nur eine durchschnittliche Anzahl von Sozialbeziehungen aufweist. Eine genaue Bezeichnung der einer Analyse zugrundeliegenden Konzepte bietet somit nicht nur der Leserin, sondern auch der Forscherin eine Möglichkeit, die Bedeutung des Konzepts in einer Menge zu verankern.

Im Weiteren werden durch die Kalibrierung von Mengen einzelnen Fällen Mitgliedschaftswerte zugeordnet, die eine Aussage über das zugrundeliegende Konzept in sich tragen. Auch hierbei gilt es, einige Fallstricke zu vermeiden, z. B. dass eine Mengenmitgliedschaft mit dem Wert 0.5 nicht vergeben werden sollte (siehe aber z. B. Braun 2013: 831; Korczynski \& Evans 2013: 775, Lee 2013: 338). Dieser Transitionspunkt bildet einen Wert, welcher genau zwischen Mitgliedschaft und Nicht-Mitgliedschaft liegt. Entsprechend wird über einen Fall mit dieser Mengenzugehörigkeit überhaupt keine Aussage getroffen, er repräsentiert das zugrundeliegende Konzept in keiner Weise - weder positiv noch negativ. ${ }^{7}$ Stattdessen sollte für jeden Fall eine Entscheidung getroffen werden, ob er nun eher Mitglied oder Nicht-Mitglied ist. „[T]o avoid the ambiguity of 0.50 " vergeben etwa Harriss-White et al. (2013: 417) in ihrer symmetrischen Kalibrierung an all diejenigen Fälle, welche in ihre Mittelkategorie gehören, den Wert 0.51 . Wenngleich dies eine eindeutige numerische Entscheidung darstellt, da nun alle diese Fälle eher Mitglied der jeweiligen Menge sind, bleibt zu beachten, dass die konzeptuelle Aussagekraft dieses Wertes nur sehr begrenzt ist und gegebenenfalls die Interpretation der Ergebnisse erschwert.

Ähnlich problematisch verhält es sich, wenn Daten, welche von vornherein in einer Ausprägung zwischen 0 und 1 vorliegen, nicht in Mengenmitgliedschaften übersetzt werden, sondern schlicht übernommen werden. So behalten z. B. Achilov und Shaykhutdinov (2013: 28) für ihre Menge ,Human Development Index (HDI) ${ }^{6}$ den Indexwert ihrer Fälle als Mengenmitgliedschaften bei. Die geringste Mengenmitgliedschaft in ,HDI' kommt demnach mit einem Wert von 0.197 Kirgisistan, der höchste Mitgliedschaftswert Albanien mit 0.319 zu. Unabhängig davon, dass somit kein Fall Mitglied in der Menge ,HDI' ist, da der Transitionspunkt (0.5) nicht überschritten wird, verfehlt eine solche Kalibrierung das Grundverständnis von Mengen. Demnach würden lediglich Fälle mit einem HDI von 1.00 eine volle Mitgliedschaft in der Menge ,HDI erhalten und nur solche eine volle Nicht-Mitgliedschaft, die einen HDI von 0.00 aufweisen. Dies ist weder konzeptuell noch empirisch sinnvoll. Darüber hinaus geht bei solch kleinteiligen Mitgliedschaftswerten bis zur dritten Nachkommastelle die qualitative Unterscheidbarkeit verloren. Mengenmitgliedschaften sollten stattdessen immer so vergeben werden, dass diese konzeptuell und empirisch begründbar sind.

In jedem Fall muss die Zuschreibung von Mitgliedschaftswerten für die Leserschaft nachvollziehbar und transparent gestaltet sein. Die einzelnen Entscheidungen über Vollmitgliedschaft, volle NichtMitgliedschaft und Transitionspunkt sollten unter keinen Umständen unerwähnt bleiben (siehe etwa Crowley 2013, 2014) oder gar einem Computer überlassen werden, um ,user-imposed biases in the analyses“ (Longest \& Thoits 2012: 204) zu vermei-

\footnotetext{
${ }^{7}$ Alle Fälle, die mit 0.5 kalibriert werden, können keiner Wahrheitstafelzeile eindeutig zugeordnet werden.
} 
den. Die Kalibrierung von Mengen ist ein qualitativer Prozess, der begründeter, transparenter Entscheidungen auf der Grundlage theoretisch-konzeptuellen Wissens und der Rückbindung an die untersuchten Fälle bedarf. Um die Entscheidungen nachvollziehen zu können, sollten sowohl die Rohdaten, d.h. die nicht kalibrierten Ausgangsdaten, als auch die kalibrierten Daten jeweils in einer Matrix im Text oder (Online-) Anhang präsentiert werden (siehe aber für viele McLevey 2014, Park 2013, Ahn \& Seung-yoon Lee 2012).

\section{Schritt 2 - Analyse notwendiger} (Kombinationen von) Bedingungen

Bei der Analyse notwendiger Bedingungen werden jede einzelne Bedingung sowie ihr jeweiliges Komplement ${ }^{8}$ betrachtet und geprüft, ob ein Outcome Y immer nur dann vorliegt, wenn auch Bedingung $X$ gegeben ist. Sollte diese Prüfung keine notwendige Bedingung hervorbringen, so besteht noch die Möglichkeit, nach verschiedenen ODER-Kombinationen (Vereinigungsmengen/unions) von Bedingungen zu suchen. Technisch ist dies auf Grundlage aller Kombinationen möglich (Thiem 2014a; Bol \& Luppi 2013). Konzeptuell ist es allerdings nur dann angeraten, wenn es sich bei den Bedingungen um funktionale Äquivalente handelt (Schneider \& Wagemann 2012: 74). So können z. B. ,hohe Bildung ODER ,hohes Erwerbseinkommen' als funktionale Äquivalente für das übergeordnete Konzepte ,hoher sozialer Status' verstanden werden. Liegen also in sämtlichen untersuchten Fälle, die das Outcome zeigen, entweder die Bedingung, hohe Bildung oder ,hohes Erwerbseinkommen' oder beides vor, kann diese Oder-Kombination nicht nur technisch einwandfrei als notwendig identifiziert, sondern auch inhaltlich interpretiert werden. Die inhaltliche Aussagekraft von notwendigen Oder-Bedingungen ist hingegen begrenzt, wenn beispielsweise in jedem Fall, der das Outcome aufweist, ,hohe Bildung' oder, in Partnerschaft ${ }^{\star}$ oder beides vorliegt, da diese beiden Bedingungen eben nicht in einem gemeinsamen, übergeordneten Konzept gefasst werden können.

In jedem Fall sollte die Analyse von notwendigen Bedingungen separat zur Prüfung auf hinreichende

\footnotetext{
${ }^{8}$ Beim Komplement einer Menge X handelt es sich um seine Negation ( X). Da Fälle in fuzzy sets partielle Mitgliedschaften aufweisen können, haben sie demnach auch eine partielle Mitgliedschaft im jeweiligen Komplement. Die Mitgliedschaft eines Falles im Komplement $\sim \mathrm{X}$ berechnet sich mittels Subtraktion des Mengenmitgliedschaftswerts $\mathrm{X}$ von 1 .
}

Bedingungen erfolgen (Schneider \& Wagemann 2010: 8; siehe allerdings für viele Blackman 2013, Ghoshal 2013, Haynes et al. 2013). Wird sie zudem der Prüfung auf hinreichende Bedingungen vorangestellt, bringt dies einige Vorteile mit sich. Erstens kann eine etwaige notwendige Bedingung in die nachfolgende Untersuchung von hinreichenden Bedingungen einbezogen werden, etwa wenn es um den Umgang mit logischen Rudimenten geht (siehe für die sogenannte enhanced standard analysis Schneider \& Wagemann 2012: 200-211). Zweitens unterliegt man durch die vorangestellte Analyse notwendiger Bedingungen nicht der Gefahr, „falsche notwendige Bedingungen" aus der Analyse hinreichender Bedingungen abzuleiten (Schneider \& Wagemann 2012: 221-226). So identifiziert bspw. Blackman (2013: 66) aus seiner Analyse hinreichender Bedingungen eine nur scheinbar notwendige Bedingung, weil diese in allen fünf hinreichenden Lösungspfaden enthalten ist (ebenso Kim 2011, Castellano 2010). Drittens wird durch die vorgelagerte Analyse notwendiger Bedingungen auch das Problem vermieden, ,versteckte notwendige Bedingungen “ zu übersehen (Schneider \& Wagemann 2012: 227-232).

\section{Schritt 3 - Analyse hinreichender (Kombinationen von) Bedingungen}

Bei der Analyse von hinreichenden Bedingungen bildet die Wahrheitstafel (truth table) "the core of QCA, both in the understanding of it as an approach and as a technique" (Schneider \& Wagemann 2012: 91). In der Wahrheitstafel werden alle logisch möglichen Kombinationen von Bedingungen abgetragen. ${ }^{9}$ Jeder Fall wird anschließend auf Grundlage seiner Mitgliedschaftswerte in den Bedingungen derjenigen Wahrheitstafelzeile zugewiesen, welche seine Bedingungskonfiguration idealtypisch wiedergibt. In einem weiteren Schritt wird über alle Fälle hinweg geprüft, ob eine Wahrheitstafelzeile, also eine idealtypische Kombination von Bedingungen, hinreichend für das Outcome ist oder nicht. Am Ende dieses Prozesses steht eine vollständige Wahrheitstafel, die anzeigt, welche Bedingungskonfigurationen als hinreichend identifiziert wurden und welche nicht. Da diese Ausdrücke jedoch recht komplex sein können, werden die als

\footnotetext{
${ }^{9}$ Sowohl bei crisp sets als auch bei fuzzy sets besteht eine Wahrheitstafel aus $2^{\mathrm{k}}$ Zeilen, wobei $\mathrm{k}$ für die Anzahl der Bedingungen steht und 2 für den möglichen Wahrheitswert der Bedingung (vorhanden oder nicht-vorhanden). Werden also drei Bedingungen betrachtet, besteht die Wahrheitstafel aus acht Zeilen, bei vier Bedingungen aus 16 usw.
} 
hinreichend identifizierten Bedingungskombinationen in einem abschließenden Analyseschritt noch vereinfacht, d. h. mit Hilfe eines auf Boolescher Algebra beruhenden Algorithmus minimiert (Schneider \& Wagemann 2012: 92-104; Ragin 2008: 124 144).

Eine Wahrheitstafel aus den kalibrierten Daten zu erstellen, stellt dank der gängigen Softwarepakete wie fsQCA oder R keine Hürde dar. Dennoch wird dieses wichtige Analyseinstrument häufig weder im eigentlichen Aufsatz noch in etwaigen (Online-) Appendizes zur Verfügung gestellt (siehe für viele Wright \& Schaffer Boudet 2012, Longest \& Thoits 2012, Crowley 2013). Dies ist nicht nur hinsichtlich mangelnder Transparenz nachteilig; es erschwert zudem, den Umgang mit begrenzter empirischer Vielfalt (limited empirical diversity) nachzuvollziehen. Diese tritt auf, wenn für eine Wahrheitstafelzeile also für eine idealtypische Bedingungskonfiguration - kein empirisch beobachtbarer Fall vorliegt (logische Rudimente, logical remainders). Dies wird am Beispiel von Wright und Schaffer Boudet (2012) besonders deutlich, die 20 Fälle untersuchen und 7 Bedingungen in ihre Analyse integrieren: 7 Bedingungen ermöglichen $2^{7}$ Bedingungskombinationen und damit 128 Wahrheitstafelzeilen. Selbst wenn also jeder Fall einen anderen Idealtypus repräsentieren würde, blieben 108 logische Rudimente die Folge. Blackman (2013) sowie Hussain und Howard (2013) untersuchen sogar jeweils 9 Bedingungen und betrachten lediglich 27 (Blackman) bzw. 20 (Hussain \& Howard) Fälle, woraus sich mindestens 485 bzw. 492 logische Rudimente ergeben. Das bedeutet, dass $94,7 \%$ der Wahrheitstafelzeilen der ersten Studie und 96,1\% der zweiten Studie ohne empirische Fälle sind.

Begrenzte empirische Vielfalt stellt deshalb ein Problem dar, weil für jede leere Wahrheitstafelzeile eine Entscheidung getroffen werden muss, ob diese potentiell auch als hinreichende Bedingung interpretiert und damit in den Minimierungsprozess einbezogen werden kann oder grundsätzlich aus der Lösung ausgeschlossen werden muss. Hierzu stehen eine Reihe unterschiedlicher Strategien zur Verfügung wie etwa die Einbeziehung etwaiger notwendiger Bedingungen, kontrafaktisches Denken (counterfactual reasoning) oder auch der Rückgriff auf theoretische Annahmen und empirische Ergebnisse anderer Studien (siehe für eine ausführliche Diskussion Schneider \& Wagemann 2012: 151176, 197-219; Ragin 2008: 147-175). In jedem Fall behauptet jedes logische Rudiment, das in die Minimierung eingebracht wird, dass wenn Fälle in dieser Merkmalskombination vorhanden wären, sie dann auch das Outcome zeigen würden. Die Entscheidung über logische Rudimente beeinflusst in ihrer Konsequenz natürlich auch die Lösungsterme einer QCA. ${ }^{10}$ Es liegt also nahe zu fordern, dass diese Entscheidungen auf einer soliden theoretischen und konzeptuellen Grundlage getroffen werden sollten. Während die mittlere (intermediate) Lösung (z. B. Lee 2013: 340) genau diese Grundlage bietet, bezieht die sparsamste (most parsimonious) Lösung (z. B. Achilov \& Shaykhutdinov 2013) automatisch alle logischen Rudimente mit ein, die das Gesamtergebnis weniger komplex machen. Die weitreichenden Implikationen dieser Entscheidung werden häufig nicht thematisiert. So argumentiert z. B. Blackman (2013: 66) „[f]or reasons of space, only the parsimonious solution for the twenty-seven cases is discussed here; it is the most interpretable and interesting." Der Software wurde in diesem Fall somit für mindestens 485(!) Wahrheitstafelzeilen überlassen zu entscheiden, ob diese hinreichend für das Outcome sind oder nicht; d. h. sie konnte aus $2^{485}$ möglichen Kombinationen diejenige wählen, welche die technisch sparsamste Lösung hervorbringt - ohne dass dies theoretischkonzeptuell rückgebunden werden konnte und ohne dass die genutzten logischen Rudimente transparent dargestellt wurden. Dass diese Lösung dann am einfachsten $\mathrm{zu}$ interpretieren ist, überrascht kaum. Grundsätzlich muss allerdings festgehalten werden, dass allen Ergebnissen, egal ob sie logische Rudimente einbeziehen oder nicht, immer die gleiche empirische Basis zugrunde liegt und sie daher alle sowohl logisch richtig sind als auch der empirischen Realität nicht widersprechen. Sie unterscheiden sich allein in ihren Annahmen bezüglich der begrenzten empirischen Vielfalt. Es ist deshalb unerlässlich, dass eine QCA den Umgang mit logischen Rudimenten so transparent wie möglich thematisiert, mögliche Strategien und Annahmen offen diskutiert und am Ende der Analyse die unterschiedlichen Lösungsterme im Spiegel des Theorieund Fallwissens der Forscherin erörtert. Es kann nicht stark genug betont werden, dass der Umgang

\footnotetext{
10 Es können grundsätzlich drei Lösungen in einer QCA unterschieden werden: die konservative Lösung (conservative solution) bezieht keine logischen Rudimente ein, während die sparsamste Lösung (most parsimonious solution) alle diejenigen logischen Rudimente in den Minimierungsprozess aufnimmt, die das Ergebnis schlanker machen. In der mittleren Lösung (intermediate solution) werden nur diejenigen logischen Rudimente in den Minimierungsprozess einbezogen, die sowohl die Lösung sparsamer machen als auch den theoretischen Erwartungen der einzelnen Bedingungen entsprechen.
} 
mit logischen Rudimenten eines der wesentlichen Gütekriterien für jede QCA darstellt.

\section{Schritt 4 - Konsistenz und Abdeckung als Gütemaße einer QCA}

In der sozialwissenschaftlichen Forschungspraxis lassen sich häufig (Kombinationen von) Bedingungen identifizieren, die nicht perfekt, d. h. nicht für sämtliche Fälle, hinreichend bzw. notwendig sind, sondern leichte bis starke Abweichungen aufweisen. Mit Konsistenz (consistency) und Abdeckung (coverage) liegen aus diesem Grund zwei Gütemaße vor, die zur Evaluierung nicht-perfekter Teilmengenbeziehungen sowohl bei notwendigen als auch hinreichenden Bedingungen herangezogen werden können (siehe zum Folgenden Schneider \& Wagemann 2012: 119-150; Ragin 2008: 44-68). Das Konsistenzmaß gibt den Gütegrad der Teilmengenbeziehungen wieder und zeigt an, wie stark sie von einer perfekten subset/superset-Beziehung abweicht. Der Abdeckungsparameter hingegen muss je nachdem, ob es sich um eine notwendige oder hinreichende Bedingung handelt, auf unterschiedliche Art und Weise interpretiert werden. Bei hinreichenden Bedingungen auf der einen Seite ist die Abdeckung ein Maßstab dafür, wie „breit“ eine Erklärung ist d.h. inwieweit ein Outcome durch eine hinreichende Bedingung erklärt werden kann. Bei notwendigen Bedingungen auf der anderen Seite zeigt die Abbildung an, wie trivial eine Bedingung ist: Ist eine Bedingung nahezu immer vorhanden, egal ob ein Outcome vorliegt oder nicht, oder ist eine Bedingungsmenge wesentlich größer als die Outcomemenge, so kann von einer trivialen notwendigen Bedingung gesprochen werden (siehe Schneider \& Wagemann 2012: 233f.).

Die beiden Parameter Konsistenz und Abdeckung leisten $\mathrm{zu}$ unterschiedlichen Phasen der Analyse wertvolle Hilfestellungen für die Bewertung der Teilmengenbeziehungen. So ist das Konsistenzmaß bei der Analyse von hinreichenden Bedingungen ein wichtiger Indikator, wenn eine Wahrheitstafelzeile nicht perfekt konsistent ist, d. h. in manchen Fällen das Outcome vorliegt und in anderen nicht. Und auch bei der Darstellung und Interpretation der Untersuchungsergebnisse von notwendigen und hinreichenden Bedingungen sind sie zwingender Bestandteil - wie z. B. bei der Diskussion äquifinaler Lösungsterme. Es ist allerdings ausdrücklich vor einer automatisierten Anwendung der Parameter zu warnen. Zwar existieren hinsichtlich akzeptabler Konsistenz- und Abdeckungsmaße bestimmte Richtwerte (z. B. durch Ragin 2009: 121 oder Schneider \& Wagemann 2012: 278-79), die jedoch nur Emp- fehlungen oder Anhaltspunkte darstellen. Die letztendliche Evaluierung muss durch die Forscherin auf Basis ihres Theoriewissens erfolgen sowie an multiplen Punkten der Analyse an die Fälle rückgekoppelt werden.

Jede Abweichung von einer perfekten hinreichenden Bedingung, d. h. jede Entscheidung, eine (leicht) inkonsistente Kombination dennoch als hinreichend für den Outcome zu bewerten, sollte daher sorgfältig und transparent begründet werden. Häufig lässt sich jedoch beobachten, dass eine solche Begründung fehlt und stattdessen auf empfohlene Grenzwerte zurückgegriffen wird (für viele Wright \& Schaffer Boudet 2012: 740), oder gar die Existenz von Grenzwerten unbelegt behauptet wird: „A threshold of 0.8 consistency ( 80 percent or higher) is generally accepted for supporting claims on necessary conditions. " (Achilov \& Shaykhutdinov 2013: 30). Eine automatisierte Anwendung der empfohlenen Grenzwerte verschleiert sowohl den Sinn der Gütemaße als auch der Empfehlungen selbst. Die oft zitierte Aussage „consistency levels (well) above 0.75 are advisable“" (Schneider \& Wagemann 2012: 279) bedeutet gerade nicht, dass 0.75 der Standardwert für die Konsistenz hinreichender Bedingungen ist. Das wäre auch dahingehend fatal, als dass jede Kombination von Bedingungen automatisch als hinreichend anzusehen wäre, sobald sie drei Viertel der abgedeckten Outcomemenge richtig beschreibt. Der Grenzwert zeigt vielmehr, dass diese Grenze keinesfalls zu unterschreiten ist. Grundsätzlich gilt, dass die Gütemaße niemals diejenigen Fälle verdecken sollten, die bspw. für eine geringe Konsistenz verantwortlich zeichnen. Stattdessen sollten diese Fälle immer identifiziert werden - bspw. mittels eines XY-Plots -, um bewerten zu können, wie stark eine Kombination von Bedingungen von einer perfekten Teilmengenbeziehung abweicht.

Ein weiteres breit auftretendes Problem der untersuchten Studien stellt die geringe Gesamtabdeckung aller äquifinalen Lösungspfade dar. QCA zielt darauf ab, ein Outcome für alle bzw. zumindest eine größtmögliche Anzahl von Fällen, in denen es auftritt, mittels verschiedener Kombinationen von Bedingungen zu erklären. Verbleibt dennoch eine geringe Abdeckung selbst einer sehr guten, d.h. konsistenten, Gesamtlösung, weist dies darauf hin, dass die gewählten Bedingungen einen Großteil des Auftretens des Outcomes nicht zu erklären vermögen. Dieses Problem wird indes häufig nicht thematisiert (z. B. Eng \& Woodside 2012), sondern es wird etwa argumentiert, eine Abdeckung von 0.43 sei „in line with many other published studies using 
fsQCA “ (Korczynski \& Evans 2013: 777). Auch wenn Abdeckungsmaße von vielerlei Faktoren abhängig sind, sollte eine höhere Abdeckung angestrebt werden und die mit einer niedrigen Abdeckung einhergehenden Probleme wiederum offen diskutiert werden.

\section{Aktuelle Anwendungsbeispiele von QCA in der Soziologie}

Im Folgenden werden drei QCA-Anwendungen vorgestellt, die die oben beschriebenen Analyseschritte nahezu umfassend durchgeführt und transparent dargestellt haben und anhand derer das Potential von QCA für soziologische Fragestellungen beispielhaft aufgezeigt werden kann. Die Auswahl der Untersuchungen versucht einerseits, eine möglichst große Bandbreite soziologischer Themen abzudecken und andererseits eine tiefergehende, detaillierte Darstellung der Studien zu gewährleisten. Aus diesem Grund wurden drei Studien ausgewählt, je eine makrosoziologische, die Nationalstaaten zum Gegenstand der Untersuchung hat, eine Studie auf der Meso-Ebene, die sich mit sozialen Gruppen auseinandersetzt, sowie eine mikrosoziologische Studie, die sich mit Individuen beschäftigt.

\section{Beispiel einer soziologischen QCA auf Makro-Ebene}

Schneider und Makszin (2014a) untersuchen in ihrer Studie „Forms of Welfare Capitalism and Education-Based Participatory Inequality“, unter welchen Bedingungen Wohlfahrtsstaaten geringe bildungsbedingte Ungleichheiten in der politischen Partizipation aufweisen. Der Frage liegt die Erwartung zugrunde, dass soziale und sozio-ökonomische Ungleichheiten sich in verschiedenen Wohlfahrtsstaatstypen unterschiedlich stark in ungleiche politische Partizipation übersetzen. Allein auf der Grundlage mikrosoziologischer Annahmen, dass „low-educated, low-income earners or other socially disadvantaged individuals tend to participate less in politics“" (Schneider \& Makszin 2014a: 438) lassen sich Länderunterschiede in der politischen Partizipation von Bürgern nämlich nicht erklären. Daher verknüpfen die Autoren theoretisch mikrofundierte Annahmen zum Einfluss von Ressourcen und Engagement auf politische Partizipation mit makrosoziologischen Theorien, nach welchen individuelle sozio-ökonomische Unterschiede durch staatliche Maßnahmen wie Arbeitsschutz sowie monetäre und nicht-monetäre Unterstützungsleistungen teilweise auszugleichen vermocht werden. Sie verzichten dabei explizit auf bestehende Typolo- gien von Wohlfahrtsstaaten und fragen, „which (combinations of) welfare capitalist traits jointly influence our dependent variable, participatory inequality. “ (Schneider \& Makszin 2014a: 440).

Empirisch verknüpfen Schneider und Makszin eine zweistufige Regression (auf der Individualebene und auf der Länderebene) mit einer fsQCA. Sie betrachten insgesamt 37 Länder zu je drei Zeitpunkten (1995, 2000, 2005). Für die QCA wählen die Autoren insgesamt 77 dieser Beobachtungen als Fälle aus. Sie untersuchen dabei die Kombinationen von vier Bedingungen, weshalb die Wahrheitstafel aus 16 idealtypischen Kombinationen von Bedingungen besteht. Da sich die 77 Fälle auf 15 Wahrheitstafelzeilen verteilen, stellt begrenzte empirische Vielfalt kaum ein Problem dar; es existiert lediglich ein einziges logisches Rudiment.

Das Outcome, low participatory inequality' ist, wie auch die vier Bedingungen, high labor market expenditure', ,high wage coordination', high union density' und, high employment protection' mit einem aussagekräftigen Label versehen. Schneider und Makszin (2014a: 449) verweisen darauf, dass der qualitative Unterschied zwischen Mitgliedschaft und Nicht-Mitgliedschaft gerade nicht in den intervall-skalierten Variablen enthalten ist und diese mengentheoretische Verankerung durch eine eigene Entscheidung erfolgen muss. Sie nutzen für sämtliche Bedingungen die direkte Kalibrierung, welche eine logarithmische Funktion über metrische Variablen legt und anhand der drei Ankerpunkte die einzelnen Mitgliedschaftswerte der Fälle berechnet. Die Autoren stellen in einem umfassenden Online-Appendix (Schneider \& Makszin 2014b: 11) die Schwellenwerte für Vollmitgliedschaft, Nicht-Mitgliedschaft und Transitionspunkt transparent dar, allerdings ohne diese qualitative Wahl eingehend zu begründen. Stattdessen führen sie einen Robustheitstest (siehe hierzu Skaaning 2011) der Kalibrierung des Outcomes durch, was als innovatives Element innerhalb einer QCA bezeichnet werden kann (Schneider \& Makszin 2014b: 19-22).

Schneider und Makszin (2014a: 451) identifizieren keine notwendige Bedingung für eine niedrige Ungleichheit in der Partizipation und verweisen auf die Berechnung, die ebenfalls im Anhang zu finden ist (Schneider \& Makszin 2014b: 14). In der Analyse hinreichender Bedingungen minimieren sie die im Text untergebrachte Wahrheitstafel zu drei hinreichenden Kombinationen von Bedingungen, welche dann zur Typologisierung von Wohlfahrtsstaatstypen genutzt werden. So identifizieren die 
Autoren eine erste Gruppe von Staaten, in denen die Kombination von starkem Arbeitsschutz für Personen mit niedrigem Bildungsstand und starker Unterstützung für Arbeitslose hinreichend für eine niedrige Ungleichheit der politischen Partizipation ist. Sie bezeichnen diesen Wohlfahrtstypus, dessen idealtypischen Vertreter Dänemark (1995) und Slowenien (1995) sind, als ,protective support‘. Darüber hinaus zeigt sich ein zweiter Wohlfahrtsstaatstypus, in welchem die Kombination aus hohen Arbeitsmarktausgaben und starken Lohnabsprachen (wage coordination) hinreichend für eine niedrige partizipatorische Ungleichheit ist. Dänemark (2000) und Irland (2000) stehen für diesen Typus des ,coordinated support' Pate. Ein dritter Wohlfahrtsstaatstypus kennzeichnet sich schließlich durch die Kombination von starkem Arbeitsschutz und schwachen Gewerkschaften. Diese Form der ,unorganized protection', wie sie Portugal (19952005), Mexiko (2000, 2005), Estland (2005) und Korea (2005) idealtypisch zeigen, ist ebenfalls hinreichend für niedrige politische Ungleichheiten.

Die Darstellung der Ergebnisse ist umfangreich und transparent. Im Text wird neben der Analyse mit Gütemaßen (nach Ragin \& Fiss 2009) auch eine Tabelle mit typischen Fällen der Wohlfahrtstypen präsentiert; der Anhang enthält zudem die XY-Plots der drei Lösungspfade und der Gesamtlösung (Schneider \& Makszin 2014b: 15-18). Während die drei Lösungspfade und die Gesamtlösung sehr hohe Konsistenzwerte aufweisen (zwischen 0.85 und 0.89), also sehr gute Erklärungen für das Outcome bieten, vermag die Lösung das Auftreten des Outcomes bei weitem nicht in Gänze zu erklären (Gesamtabdeckung der Lösung 0.58). Schneider und Makszin (2014a: 459) diskutieren diesen Punkt offen und verweisen in ihrem Fazit darauf, dass weitere Wohlfahrtstypen durch ihre Auswahl von immerhin nur vier - Bedingungen unberücksichtigt geblieben sind.

\section{Beispiel einer soziologischen QCA auf der Meso-Ebene}

Cebotari und Vink (2013) untersuchen in ihrem Artikel „A Configurational Analysis of Ethnic Protest in Europe" 29 ethnische Minderheiten in Europa hinsichtlich der Frage, unter welchen Bedingungen diese starkes bzw. schwaches Protestverhalten aufweisen. Sie kritisieren bestehende, vornehmlich quantitative Studien zum einen dahingehend, dass deren Befunde auf eine große Fallzahl auch stark unterschiedlicher ethnischer Minderheiten generalisiert werden würden. Zum anderen würden bestehende Studien aufgrund ihrer additiven Regres- sionsmodelle behaupten, dass die gefundenen „causal conditions can independently explain whether groups are more or less mobilized" (Cebotari \& Vink 2013: 298). Die beiden Autoren verweisen daher auf die Vorteile der kombinatorischen Herangehensweise von QCA und interessieren sich für verschiedene Erklärungspfade, die das Protestverhalten unterschiedlicher ethnischer Minderheiten fallorientiert erklären. Theoretisch verknüpfen Cebotari und Vink ethnopolitisch orientierte Konfliktliteratur mit den Grundlagen politischer Partizipationsforschung und definieren die Mobilisierung zu ethnisch motivierten Protesten als „non-violent form of contentious politics" (Cebotari \& Vink 2013: 301). Um die unterschiedlichen Ausformungen von schwachem und starkem gewaltlosem Protest erklären zu können, integrieren sie drei gruppenbezogene und zwei institutionelle Bedingungen in den Analyserahmen. Einer hohen ,Demokratiestärke' wird zugeschrieben, einen friedlichen Protest zu ermöglichen, einer starken, ethnischen Zersplitterung' als zweiter institutioneller Bedingung hingegen, hinreichend, aber nicht notwendig für starken Protest zu sein. Bei den gruppenbezogenen Bedingungen handelt es sich (1) um hohe ,geographische Konzentration', welche die Kapazität beschreibt, einen wirkungsvollen Protest überhaupt zu organisieren, (2) um hohe, politische Diskriminierung', welche der jeweiligen ethnischen Minderheit widerfährt, und als Impuls für Protest dienen kann sowie (3) um starken, Nationalstolz' innerhalb der Minderheit als Proxy für eine nationale Identität, die starke Proteste unwahrscheinlich macht.

Die Autoren stellen die Kalibrierung ihrer Mengen recht ausführlich dar. Im Anhang I des Artikels beschreiben sie neben der jeweiligen Datenquelle jeweils eine „brief elaboration of calibration“ und geben die qualitativen Ankerpunkte für Voll- und Nichtmitgliedschaft sowohl Transitionspunkt an (Cebotari \& Vink 2013: 319-321). Es fällt auf, dass sie die Daten jeweils symmetrisch kalibrieren, um im Weiteren die Analyse von ,starkem Protest ${ }^{\star}$ und ,schwachem Protest' mit den gleichen Mengenmitgliedschaften durchführen zu können. Aussagekräftige Labels vergeben Cebotari und Vink indes nicht; die Bedingungen heißen ,democracy', ,ethnic fractionalization', ,geographical concentration', ,political discrimination" und ,national pride‘.

Aus den fünf Bedingungen ergeben sich 32 logisch mögliche Kombinationen. Neben den drei arithmetischen Rudimenten (32 Wahrheitstafelzeilen - 29 Fälle) stellen mehrere Fälle den gleichen Idealtypus dar, weshalb nur 17 der 32 Idealtypen mit Fällen abgedeckt werden. Obschon die Autoren die spar- 
samste Lösung anwenden, gehen sie transparent mit der Nutzung der 15 logischen Rudimente um (Cebotari \& Vink 2013: 315).

Die Analyse der Mengenbeziehungen führen Cebotari und Vink sowohl ausführlich als auch in der oben beschriebenen Reihenfolge aus. Für ,starken Protest' identifizieren sie, geographische Konzentration' als notwendige Bedingung, für ,schwachen Protest' hingegen ,Nationalstolz'. Da es sich nicht um perfekt konsistente notwendige Bedingungen handelt, verweisen sie einerseits auf den „standard consistency threshold of 0.9 " (Cebotari \& Vink 2013: 307) von Ragin (2009) und Schneider \& Wagemann (2012). Andererseits erinnern sie an die inhaltliche Bedeutung nicht-perfekter Konsistenz und sprechen daher im Weiteren nur von „quasi-necessary conditions" (Ragin 2000).

Die (sparsamste) Analyse hinreichender Bedingungen für das Outcome ,starker Protest' bringt vier Kombinationen von Bedingungen hervor, die das Auftreten des Outcomes insgesamt sehr gut (Konsistenz 0.88) und sehr breit (Abdeckung 0.81) erklären. Interessant ist hierbei, dass nur drei der vier Pfade die quasi-notwendige Bedingung, geographische Konzentration' aufweisen. Es handelt sich dabei um die Kombination von ,geographischer Konzentration' mit entweder den beiden institutionellen Bedingungen ,Demokratiestärke' UND ,ethnischer Zersplitterung', ODER mit ,ethnischer Zersplitterung' UND ,politischer Diskriminierung ODER mit ,politischer Diskriminierung ' UND ,Demokratiestärke‘. Der vierte Pfad besteht nur aus der Abwesenheit von ,Nationalstolz' (Cebotari \& Vink 2013: 309).

Auch die (sparsamste) Analyse, schwachen Protests' weist sehr hohe Werte auf (Konsistenz 0.82, Abdeckung 0.89). Die ebenfalls vier Kombinationen enthalten die notwendige Bedingung ,Nationalstolz'. Hinreichend wird diese Bedingung in Kombination mit entweder der Abwesenheit von ,politischer Diskriminierung' UND der Abwesenheit von ,ethnischer Zersplitterung' ODER mit der Abwesenheit von ,politischer Diskriminierung' UND der Abwesenheit von ,Demokratiestärke‘ ODER mit der Abwesenheit von ,Demokratiestärke' UND der Abwesenheit von ,ethnischer Zersplitterung' ODER der Abwesenheit von ,geographischer Konzentration'. Die Darstellung dieser sprachlich nur recht komplex formulierbaren Ergebnisse erfolgt in einer Tabelle anhand aller Fälle, die das Outcome aufweisen (Cebotari \& Vink 2013: 309, 311), jedoch ohne die graphische Unterstützung von XY-Plots. Das Papier endet mit der Einordnung von QCA und der Ergebnisse in das Forschungsfeld und möglicher methodisch und inhaltlich weiterführender Designs. Eine Rückbindung der Ergebnisse an einzelne Fälle bleibt indes leider aus.

\section{Beispiel einer soziologischen QCA auf der Mikro-Ebene}

Marr (2012) untersucht in seinem Artikel „Pathways out of Homelessness in Los Angeles and Tokyo: Multilevel Contexts of Limited Mobility amid Advanced Urban Marginality" das Phänomen der Massenobdachlosigkeit in Großstädten der Welt. Er fragt, unter welchen Kombinationen von ökonomischen, organisationalen und individuellen Bedingungen Wege aus der Obdachlosigkeit gefunden werden können. Marr (2012) untersucht dazu 34 Fälle - jeweils 17 Individuen, welche in Übergangsunterkünften in Tokyo und Los Angeles mehrfach interviewt wurden. Wie auch das Outcome ,exited', welches den Wohnzustand der Fälle 30 Tage nach dem Verlassen der Übergangsunterkünfte in eine fuzzy Menge transformiert, sind auch die vier Bedingungen sehr transparent und mit je vier qualitativ klar unterscheidbaren fuzzy-Werten (Vollmitgliedschaft 1, eher Mitglied als Nicht-Mitglied 0.67, eher Nicht-Mitglied als Mitglied 0.33 und NichtMitglied 0) kalibriert (Marr 2012: 991). Die Menge ,minimum wage' gibt als ökonomische Rahmenbedingung den finanziellen Spielraum der untersuchten Personen an. Die Hypothese dazu lautet, dass ein Zugang zum Niedriglohn-Arbeitsmarkt in Tokyo den Ausstieg aus der Obdachlosigkeit weitaus stärker ermöglicht als eine Arbeitsstelle im Niedriglohnsektor in Los Angeles. Die Bedingung ,staff ally' deckt den organisationalen Einfluss ab und betrachtet, wie intensiv und konfliktbehaftet die Beziehung der untersuchten Personen mit dem Personal der Übergangsunterkunft und den örtlichen Behörden verlief. Die individuelle Bedingung ,social tie', beschreibt, wie intensiv persönliche Kontakte mit nicht-obdachlosen Freunden und Verwandten gehalten werden. Folgende Hypothese wird zu dieser Bedingung formuliert. Demnach erwartet der Autor, dass Personen mit starken sozialen Beziehungen in Los Angeles aufgrund der kulturellen Gegebenheiten wesentlich leichter der Obdachlosigkeit entrinnen als ebensolche Personen in Tokyo. Der Bedingung, recently dislocated', welche persönliche Erfahrungen wie physische oder psychische Erkrankungen, frühere Phasen von Obdachlosigkeit und ähnliches umfasst, wird schließlich in beiden Städten ein geringerer Einfluss auf den Ausstieg aus der Obdachlosigkeit zugeschrieben als den ökonomischen und sozialen Bedingungen. 
In der weiterführenden Analyse clustern die Fälle in Los Angeles stark und bilden nur fünf der 16 Idealtypen empirisch ab (11 logische Rudimente); in Tokyo hingegen werden neun der 16 Bedingungskombinationen abgedeckt (7 logische Rudimente). Während eine Analyse notwendiger Bedingungen ausbleibt, beruht die Analyse hinreichender Bedingungen auf der mittleren (intermediate) Lösung. Marr (2012: 993) legt transparent dar, nach welchen theoretischen Erwartungen er die logischen Rudimente auswählt, die seine Lösungspfade schmaler machen. Die Ergebnisse beider Analysen, Los Angeles und Tokyo, präsentiert er in einer Tabelle, die auch die Gütemaße umfasst. In Los Angeles vermag von den zwei hinreichenden Kombinationen (Gesamtkonsistenz 0.77, -abdeckung 0.84) insbesondere die Verbindung von, staff ally' UND ,social tie' einen großen Anteil des Outcomes zu erklären (Abdeckung dieses Pfades 0.5). Erwartungsgemäß sind es somit auch empirisch vor allem die sozialen Erklärungsfaktoren, welche den Ausstieg aus der Obdachlosigkeit in Los Angeles ermöglichen. Der zweite Lösungspfad umfasst ebenfalls die Bedingung ,social tie ${ }^{6}$, allerdings in Kombination mit ,minimum wage', also einer Arbeit im Niedriglohnbereich UND ,recently dislocated', also früheren Erfahrungen mit Obdachlosigkeit. Auch für die befragten Personen in Tokyo identifiziert Marr (2012: 994) zwei gut und breit erklärende hinreichende Pfade (Gesamtkonsistenz 0.93, -abdeckung 0.93), von denen einer allein aus der Bedingung, minimum wage' besteht (Abdeckung dieses Pfades 0.6). Erwartungsgemäß kommt der ökonomischen Bedingung somit eine starke Erklärungskraft zu. Der zweite Pfad besteht aus der Kombination aus früheren Erfahrungen mit Obdachlosigkeit (,recently dislocated') UND der Abwesenheit sozialer Bindungen (, social ties`). Abschließend bindet Marr (2012) die Ergebnisse an die theoretischen und konzeptuellen Überlegungen rück.

\section{Zusammenfassend-vergleichende Betrachtung der drei Anwendungen}

In der vergleichenden Analyse der drei Studien auf Makro-, Meso- und Mikroebene lässt sich im Wesentlichen dreierlei erkennen. Zum einen zeigt sich die Vielfalt der möglichen Anwendungen und $\mathrm{Da}$ tenquellen, aus welchen Mengen kalibriert werden können, als eine zentrale Stärke von QCA. Während die ersten beiden Studien vornehmlich quantitative Indikatoren nutzen und durch qualitative Entscheidungen in aussagekräftige Mengen transformieren, die das zugrundeliegende (teilweise sogar asymmetrische) Konzept repräsentieren, nutzt
Marr (2012) auch Interviewdaten, Krankenakten und Informationen aus Lebensläufen, um seine Mengen zu kalibrieren. Zum zweiten nutzen alle drei Studien die Methode QCA mit großer Sorgfalt und im Wissen um ihre Probleme und Fallstricke. Mit beträchtlichem Aufwand werden qualitative Entscheidungen transparent und umfassend begründet sowie Gütemaße und der häufig schwierige Umgang mit logischen Rudimenten besprochen. Dennoch zeigt sich zum dritten, dass selbst die hier als best practices vorgestellten Studien nicht perfekt sind. Schneider und Makszin (2014a) legen die Ankerpunkte ihrer Kalibrierung offen, begründen jedoch nicht, warum sie diese gewählt haben. Cebotari und Vink (2013) identifizieren aus ihren Daten je eine (quasi-)notwendige Bedingung für das Auftreten und Nicht-Auftreten des Outcomes, nutzen diese Information jedoch nicht, um ihre logischen Rudimente für die sparsamste Lösung auszuwählen (siehe Schneider \& Wagemann 2012: 200-211). Marr (2012) analysiert schließlich notwendige Bedingungen überhaupt nicht. Auch dass drei Studien bei gleicher methodischer Herangehensweise drei unterschiedliche Formen der Darstellung ihrer Ergebnisse wählen, ist auf den ersten Blick suboptimal. Positiv gewendet, bieten die Studien somit eine Übersicht über die gängigsten Formen der Ergebnissicherung von QCAs: (1) eine einfache Tabelle der minimierten Lösungsterme (Marr 2012: 994), (2) eine Tabelle mit den Mitgliedschaften jedes das Outcome aufweisenden Falles in jeder hinreichenden Kombination von Bedingungen (Cebotari \& Vink 2013: 209) oder (3) die von Ragin und Fiss (2009) empfohlene Darstellung von Kern- und peripheren Bedingungen, welche um XY-Plots erweitert wird (Schneider \& Makszin 2014a: 452, 2014b: 15-18). Zudem weisen alle drei Darstellungsformen die wichtigsten Informationen auf - es werden sowohl die einzelnen Lösungspfade und die komplette Lösung mit ihren Gütemaßen korrekt wiedergegeben als auch eine Rückbindung an die untersuchten Fälle ermöglicht.

\section{Abschließende Bemerkungen zum Mehrwert von QCA für die Soziologie}

Zielsetzung dieses Beitrags war es, den originären Mehrwert sowie Forschungsperspektiven und -potentiale von QCA für soziologische Frage- und Themenstellungen am Beispiel von publizierten Journalartikeln darzulegen. Dabei sind drei Aspekte besonders hervorzuheben:

Erstens unterscheiden sich mengentheoretische Ansätze wie QCA grundlegendend von korrelativen 
Analyseverfahren - sei es in ihren zentralen Bausteinen der Analyse wie Mengen vs. Variablen, oder in ihren Funktionslogiken. So eröffnet die Suche nach notwendigen und hinreichenden Bedingungen einen gänzlich anderen Blick auf soziale Phänomene als beispielsweise etablierte statistische Methoden. Goertz und Mahoney (2013) sprechen in diesem Zusammenhang auch von einem methodologischen Rorschach-Test. Dabei kann die Einnahme einer mengentheoretischen Perspektive gegebenenfalls Erklärungszusammenhänge aufdecken, welche nicht durch Korrelationen erfasst werden (siehe Ragin 2008; Goertz \& Mahoney 2012). Darüber hinaus ist QCA mit seinem grundlegenden Verständnis besonders sensitiv gegenüber Diversität wie beispielsweise äquifinaler Erklärungsfaktoren, INUSund SUIN-Bedingungen oder asymmetrischer Wirkungszusammenhängen. Insofern sollte QCA eine mehr als willkommene Erweiterung des sozialwissenschaftlichen Methodenkanons darstellen.

Methodologisch bietet QCA, zweitens, einen besonderen Zugang zu mixed-und multi-methods Designs (vgl. u. a. Ebbinghaus 2014: 365; Hollstein 2014). Zum einen kann - und sollte - QCA als mengentheoretischer Ansatz durchaus mit anderen Methoden kombiniert werden. Studien wie beispielsweise die Arbeiten von Schneider und Makszin (2014a) oder Hollstein und Wagemann (2014) verdeutlichen auf innovative Art und Weise, wie unterschiedliche methodische Ansätze wie etwa Regressionen oder Netzwerkanalysen mit QCA verknüpft werden können. Weitere Möglichkeiten zur Verbindung von QCA mit anderen methodischen Herangehensweisen stellen detaillierte Einzelfallstudien oder process tracing zur Aufdeckung von Kausalmechanismen dar; hierzu existieren einige Vorschläge für eine systematische Fallauswahl im Anschluss an eine QCA (grundlegend Rohlfing \& Schneider 2013; Schneider \& Rohlfing 2013, 2014). Zum anderen kann QCA selbst als mixed-methods Ansatz verstanden werden (siehe insbesondere Hollstein 2014: 17). Während Elemente wie Kalibrierung und Konzeptformierung, iteratives Hin-und-Her zwischen Theorie und Empirie, konfiguratives Denken oder die Fallorientierung das Qualitative in QCA betonen, unterstreichen Merkmale wie der systematische Fallvergleich selbst mittlerer und größerer Fallzahlen, die Transformation von Informationen in Zahlen (sogenannte dataset observations; siehe Brady \& Collier 2004), oder der Einsatz von mathematischen Regeln, Formeln und Parametern das Quantitative in QCA. ${ }^{11}$ Ragin

11 Gerade den deutschsprachigen Sozialwissenschaften, in denen qualitatives Arbeiten in der Regel mit interpreta-
(1987) selbst hat QCA deshalb schon früh als „dritten Weg" zwischen quantitativer und qualitativer Forschung bezeichnet (siehe auch Wagemann 2015). ${ }^{12}$

In diesem Sinne könnte eine stärkere Berücksichtigung von QCA, drittens, auch die allgemeine methodologische Diskussion in den Sozialwissenschaften bereichern. So werden etwa in der quantitativ ausgerichteten Soziologie aktuell Diskussionen geführt, die zahlreiche konzeptuelle Schnittmengen mit QCA aufzeigen, wie zum Beispiel die Argumentationslogiken kontrafaktischer Kausalinferenz von Morgan und Winship (2007). Schließlich würde umgekehrt auch QCA von einem konstruktiven methodologischen Dialog profitieren, da grundlegende Probleme wie Transparenz, Reliabilität und Validität, Visualisierung, Robustheit und Interpretation von Ergebnissen einen gemeinsamen Problemhorizont aller Methoden darstellen. Bestes Beispiel ist hier sicherlich die gegenwärtige (scharf geführte) Debatte um den (Un-)Sinn von Simulationen als Robustheitschecks bei QCAs (einführend siehe Rohlfing 2015). ${ }^{13}$

Aus einer inhaltlichen Perspektive kann festgehalten werden, dass die Rundschau der publizierten Studien das Potential von QCA für die Soziologie deutlich offengelegt hat. So kann mittels QCA eine Vielfalt unterschiedlicher Themen- und Fragestellungen untersucht werden, gleich ob auf Mikro-, Meso- oder Makro-Ebene. Insbesondere zeigt sich, dass sowohl qualitative Informationen als auch quantitative Daten in die Analyse integriert werden können. Darüber hinaus konnte herausgestellt werden, dass QCA keineswegs auf mittlere Fallzahlen beschränkt ist, sondern durchaus auch bei large-N Designs angewendet werden kann. Entscheidendes Kriterium für eine QCA ist vielmehr das oben beschriebene Grundverständnis von QCA als mengen-

tiv-hermeneutischen bzw. Sinn-verstehenden Methoden gleichgesetzt wird (vgl. etwa Hollstein \& Ullrich 2003; Lamnek 2010), dürfte es folglich nicht unbedingt leicht fallen, das Q in QCA als qualitativ anzuerkennen.

${ }^{12} \mathrm{Im}$ Französischen findet sich auch das Akronym AQQC, das für Analyse Quali-Quantitative Comparée steht (u. a. bei DeMeur und Rihoux 2002). An anderer Stelle wird sogar ganz auf das Q verzichtet und stärker das konfigurative und fallorientierte Grundverständnis in den Vordergrund der Methode gestellt (vgl. Rihoux \& Ragin 2009; Hall 2003; Blatter \& Haverland 2012).

${ }^{13}$ Für einen weiterführenden Einstieg in die Diskussion siehe Lucas \& Szatrowski 2014; Collier 2014; Ragin 2014; Seawright 2014; Vaisey 2014; Krogslund \& Michel 2014; Krogslund et al. 2015; Hug 2013, 2014; Thiem 2014b; Schneider \& Rohlfing 2014. 
theoretischem und fallorientiertem Ansatz zur Analyse von notwendigen und hinreichenden Bedingungskonfigurationen.

\section{Literatur}

Achilov, D. \& R. Shaykhutdinov, 2013: State Regulation of Religion and Radicalism in the Post-Communist Muslim Republics. Problems of Post-Communism 60: 17-33.

Adcock, R. \& D. Collier, 2001: Measurement Validity: A Shared Standard for Qualitative and Quantitative Research. American Political Science Review 95: 529546.

Ahn, S.-H. \& S. Seung-yoon Lee, 2012: Explaining Korean Welfare State Development with New Empirical Data and Methods. Asian Social Work and Policy Review 6: 67-85.

Amenta, E., N. Caren, S.J. Olasky \& J.E. Stobaugh, 2009: All the Movements Fit to Print: Who, What, When, Where, and Why SMO Families Appeared in the New York Times in the Twentieth Century. American Sociological Review 74: 636-656.

Baltzer, M, H. Westerlund, M. Backhans \& K. Melinder, 2011: Involvement and Structure: A Qualitative Study of Organizational Change and Sickness Absence among Women in the Public Sector in Sweden. BMC Public Health 11: 318-334.

Bauwens, J., B. Lobe, K. Segers \& L. Tsaliki, 2009: A Shared Responsibility. Journal of Children and Media 3: 316-330.

Bentele, K.G., 2013: Distinct Paths to Higher Inequality? A Qualitative Comparative Analysis of Rising Earnings Inequality among U.S. States, 1980-2010. Research in Social Stratification and Mobility 34: 30-57.

Blackman, T., 2008: Can Smoking Cessation Services be Better Targeted to Tackle Health Inequalities? Evidence from a Cross-Sectional Study. Health Education Journal 67: 91-101.

Blackman, T., 2013: Exploring Explanations for Local Reductions in Teenage Pregnancy Rates in England: An Approach Using Qualitative Comparative Analysis. Social Policy and Society 12: 61-72.

Blackman, T. \& K. Dunstan, 2010: Qualitative Comparative Analysis and Health Inequalities: Investigating Reasons for Differential Progress with Narrowing Local Gaps in Mortality. Journal of Social Policy 39: 359-373.

Blackman, T., J. Wistow \& D. Byrne, 2011: A Qualitative Comparative Analysis of Factors Associated with Trends in Narrowing Health Inequalities in England. Social Science \& Medicine 72: 1965-1974.

Blatter, J. \& M. Haverland, 2012: Designing Case Studies. Explanatory Approaches in Small-N Research. Houndmills, Basingstoke: Palgrave Macmillan.

Bleijenbergh, I. \& C. Roggeband, 2007: Equality Machineries Matter: The Impact of Women's Political Pres- sure on European Social-Care Policies. Social Politics: International Studies in Gender, State and Society 14: 437-459.

Bol, D. \& F. Luppi, 2013: Confronting Theories Based on Necessary Relations: Making the Best of QCA Possibilities. Political Research Quarterly 66: 205-210.

Brady, H.E. \& D. Collier (Hrsg.), 2004: Rethinking Social Inquiry. Diverse Tools, Shared Standards. Lanham: Rowman and Littlefield.

Braun, C., 2013: The Driving Forces of Stability: Exploring the Nature of Long-Term Bureaucracy-Interest Group Interactions. Administration \& Society 45: 809-836.

Britt, D.W. \& M.I. Evans, 2007: Sometimes Doing the Right Thing Sucks: Frame Combinations and Multi-Fetal Pregnancy Reduction Decision Difficulty. Social Science \& Medicine 65: 2342-2356.

Castellano, J.M., 2010: Secondary Education and Broadband Diffusion: A Qualitative Comparative Analysis. Info 12: 121-138.

Cebotari, V. \& M.P. Vink, 2013: A Configurational Analysis of Ethnic Protest in Europe. International Journal of Comparative Sociology 54: 298-324.

Collier, D., 2014: Comment: QCA Should Set Aside the Algorithms. Sociological Methodology 44: 122-126.

Crowley, M., 2014: Class, Control, and Relational Indignity: Labor Process Foundations for Workplace Humiliation, Conflict, and Shame. American Behavioral Scientist 58: 416-434.

Crowley, M., 2013: Gender, the Labor Process and Dignity at Work. Social Forces 91: 1209-1238.

Crowley, M, 2012: Control and Dignity in Professional, Manual and Service-Sector Employment. Organization Studies 33: 1383-1406.

Da Roit, B. \& B. Weicht, 2013: Migrant Care Work and Care, Migration and Employment Regimes: A FuzzySet Analysis. Journal of European Social Policy 23: 469-86.

DeMeur, G. \& B. Rihoux, 2002: L'analyse quali-quantitative comparée (AQQC-QCA). Louvain-La-Neuve: Academia Bruylant.

Ebbinghaus, B., 2014: Renzension G. Goertz / J. Mahoney (2012): A Tale of Two Cultures. Qualitative and Quantitative Research in the Social Sciences. Soziologische Revue 37: 363-365.

Eng, S. \& A.G. Woodside, 2012: Configural Analysis of the Drinking Man: Fuzzy-Set Qualitative Comparative Analyses. Addictive Behaviors 37: 541-543.

Epple, R., M. Gasser, S. Kersten, M. Nollert \& S. Schief, 2014: Institutions and Gender Time Inequality: A Fuzzy-Set QCA of Swiss Cantons. Swiss Journal of Socio$\operatorname{logy} 40: 259-278$.

Ghoshal, R.A., 2013: Transforming Collective Memory: Mnemonic Opportunity Structures and the Outcomes of Racial Violence Memory Movements. Theory and Society 42: 329-350.

Giugni, M. \& A. Nai, 2013: Paths Towards Consensus: Explaining Decision Making within the Swiss Global Justice Movement. Swiss Political Science Review 19: 26-40. 
Giugni, M. \& S. Yamasaki, 2009: The Policy Impact of Social Movements: A Replication Through Qualitative Comparative Analysis. Mobilization: An International Quarterly 14: 467-484.

Glaesser, J. \& B. Cooper, 2011: Selectivity and Flexibility in the German Secondary School System: A Configurational Analysis of Recent Data from the German SocioEconomic Panel. European Sociological Review 27: 570-585.

Glaesser, J. \& B. Cooper, 2012a: Educational Achievement in Selective and Comprehensive Local Education Authorities: A Configurational Analysis. British Journal of Sociology of Education 33: 223-244.

Glaesser, J. \& B. Cooper, 2012b: Gender, Parental Education, and Ability: Their Interacting Roles in Predicting GCSE Success. Cambridge Journal of Education 42: 463-480.

Goertz, G, 2006: Social Sciences Concepts. A User's Guide. Princeton: Princeton University Press.

Goertz, G. \& J. Mahoney, 2012: A Tale of Two Cultures: Qualitative and Quantitative Research in the Social Sciences. Princeton: Princeton University Press.

Goertz, G. \& J. Mahoney, 2013: Methodological Rorschach Tests: Contrasting Interpretations in Qualitative and Quantitative Research. Comparative Political Studies 46: 236-251.

Grant, D., M.N. Trautner, L. Downey \& L. Thiebaud, 2010: Bringing the Polluters back in: Environmental Inequality and the Organization of Chemical Production. American Sociological Review 75: 479-504.

Grant, D., A. Morales \& J.J. Sallaz, 2009: Pathways to Meaning: A New Approach to Studying Emotions at Work. American Journal of Sociology 115: 327-364.

Grant, D., K. O’Neil \& L. Stephens, 2009: Pluralistic Ignorance Among Assembled Peers. Sociological Perspectives 52: 59-79.

Hafner-Fink, M., B. Malnar \& S. Uhan, 2013: The National Contexts of Post-National Citizenship. Czech Sociological Review 49: 867-901.

Hall, P.A., 2003: Aliging Ontology and Methodology in Comparative Research. S. 373-404 in: J. Mahoney \& D. Rueschemeyer (Hrsg.), Comparative Historical Analysis in the Social Sciences. Cambridge: Cambridge University Press.

Harriss-White, B., W. Olsen, P. Vera-Sanso, \& V. Suresh, 2013: Multiple Shocks and Slum Household Economies in South India. Economy and Society 42: 398-429.

Haynes, P., 2011: Are Scandinavian Countries Different? A Comparison of Relative Incomes for Older People in OECD Nations. Social Policy \& Administration 45: 114-130.

Haynes, P., L. Banks \& M. Hill, 2013: Social Networks amongst Older People in OECD Countries: A Qualitatative [sic] Comparative Analysis. Journal of International and Comparative Social Policy 29: 15-27.

Haynes, P., M. Hill \& L. Banks, 2010: Older People’s Family Contacts and Long-term Care Expenditure in OECD Countries: A Comparative Approach Using Qualitative Comparative Analysis. Social Policy \& Administration 44: 67-84.
Hodson, P., V.J. Roscigno \& S.H. Lopez, 2006: Chaos and the Abuse of Power. Work and Occupations 33: 382-416.

Hörisch, F., 2012: Die Bestimmungsfaktoren der Einführung der Unternehmensmitbestimmung in den OECDStaaten - Eine fuzzy-set Qualitative Comparative Analysis. Zeitschrift für Sozialreform 58: 33-57.

Hollstein, B., 2014: Mixed Methods Social Networks Research: An Introduction. S. 3-34 in Domínguez, S. \& B. Hollstein (Hrsg.), Mixed Methods Social Networks Research: Design and Applications. Cambridge: Cambridge University Press.

Hollstein, B. \& C.G. Ullrich, 2003: Einheit trotz Vielfalt? Zum konstitutiven Kern qualitativer Forschung. Soziologie 32: 29-43.

Hollstein, B. \& C. Wagemann, 2014: Fuzzy-Set Analysis of Network Data as Mixed Method: Personal Networks and the Transition from School to Work. S. 237-268 in: S. Domínguez \& B. Hollstein (Hrsg.), Mixed Methods Social Networks Research: Design and Applications. Cambridge: Cambridge University Press.

Hug, S., 2013: Qualitative Comparative Analysis: How Inductive Use and Measurement Error Lead to Problematic Inference. Political Analysis 21: 252-265.

Hug, S., 2014: We Need an Open Discussion of QCA's Limitations: A Comment on Thiem. Qualitative and Multi-Method Research, Newsletter of the APSA Section for Qualitative and Multi-Method Research 12: 24-27.

Hussain, M.M. \& P.N. Howard, 2013: What Best Explains Successful Protest Cascades? ICTs and the Fuzzy Causes of the Arab Spring. International Studies Review 15: 48-66.

Ignatow, G., 2011: What has Globalization Done to Developing Countries' Public Libraries? International Sociology 26: 746-768.

Ishida, A., M. Yonetani \& K. Kosaka, 2006: Determinants of Linguistics Human Rights Movements: An Analysis of Multiple Causation of LHRs Movements Using a Boolean Approach. Social Forces 84: 19381955 .

Jang, D.-H., 2009: Significance of Variations between Income Transfers and Social Care Services Development. Journal of Comparative Social Welfare 25: 37-48.

Kim, S.H., 2011: On the Historical Determinants of Third Sector Strength: A Qualitative Comparative Analysis. Social Science Journal 48: 641-650.

Korczynski, M. \& C. Evans, 2013: Customer Abuse to Service Workers: An Analysis of its Social Creation within the Service Economy. Work, Employment \& Society 27: 768-784.

Kröger, M., 2011: Promotion of Contentious Agency as a Rewarding Movement Strategy: Evidence from the MST-Paper Industry Conflicts in Brazil. Journal of Peasant Studies 38: 435-458.

Krogslund, C., D.D. Choi \& M. Poertner, 2015: Fuzzy Sets on Shaky Ground: Parameter Sensitivity and Confirmation Bias in fsQCA. Political Analysis 23: 21-41.

Krogslund, C. \& K. Michel, 2014: A Larger-N, Fewer Variables Problem? The Counterintuitive Sensitivity of 
QCA. Qualitative and Multi-Method Research. Newsletter of the APSA Section for Qualitative and MultiMethod Research 12: 25-32.

Lamnek, S., 2010: Qualitative Sozialforschung. Weinheim: Beltz.

Laux, T., 2015: Nationalstaatliche Prozesse oder globale Strukturen? Eine Analyse der Mechanismen zur Gleichstellung von Frauen im Recht. Berliner Journal für Soziologie 24: 531-558.

Lee, S.S.-Y, 2013: High Non-Standard Employment Rates in the Republic of Korea and Japan: Analyzing Policy Configurations with Fuzzy-Set/QCA. Policy and Society 32: 333-344.

Longest, K.C. \& P.A. Thoits. 2012: Gender, the Stress Process, and Health: A Configurational Approach. Society and Mental Health 2: 187-206.

Lucas, S.R. \& A. Szatrowski, 2014: Qualitative Comparative Analysis in Critical Perspective. Sociological Methodology 44: 1-79.

Mackie, J.L., 1974: The Cement of the Universe. Oxford: Oxford University Press.

Mahoney, J., E. Kimball \& K.L. Koivu, 2009: The Logic of Historical Explanation in the Social Sciences. Comparative Political Studies 42: 114-46.

Marr, M.D., 2012: Pathways out of Homelessness in Los Angeles and Tokyo: Multilevel Contexts of Limited Mobility amid Advanced Urban Marginality. International Journal of Urban and Regional Research 36: 980-1006.

Marx, A., B. Rihoux \& C.C. Ragin, 2014: The Origins, Development, and Application of Qualitative Comparative Analysis: The First 25 Years. European Political Science Review 6: 115-142.

McAdam, D., H.S. Boudet, J. Davis, R.J. Orr, W.R. Scott \& R.E. Levitt, 2010: Site Fights: Explaining Opposition to Pipeline Projects in the Developing World. Sociological Forum 25: 401-427.

McLevey, J., 2014: Think Tanks, Funding, and the Politics of Policy Knowledge in Canada. Canadian Review of Sociology 51: 54-75.

McVeigh, R., C. Neblett \& S. Shafiq, 2006: Explaining Social Movement Outcomes: Multiorganizational Fields and Hate Crime Reporting. Mobilization: An International Journal 11: 23-49.

Melinder, K.A., 2007: Socio-Cultural Characteristics of High versus Low Risk Societies Regarding Road Traffic Safety. Safety Science 45: 397-414.

Morgan, S.L. \& C. Winship, 2007: Counterfactuals and Causal Inference. Methods and Principles for Social Research. Cambridge: Cambridge University Press.

Moritz, M., J. Giblin, M. Ciccone, A. Davis, J. Fuhrman, M. Kimiaie, S. Madzsar, K. Olson \& M. Senn, 2011: Social Risk-Management Strategies in Pastoral Systems: A Qualitative Comparative Analysis. Cross-Cultural Research 45: 286-317.

Park, S.H., 2013: Capital Openness, Monetary Integration, and Wage-Setting Coordination in Developed European Countries. Economic and Industrial Democracy 34: 637-666.

Perry, A.M. \& M.J. Schafer, 2014: Resilience in Louisiana
FEMA Parks: A Person-Centered, Fuzzy-Set Analysis. Sociological Spectrum 34: 39-60.

Ragin, C.C., 1987: The Comparative Method. Moving Beyond Qualitative and Quantitative Strategies. Berkley: University of California Press.

Ragin, C.C., 1994: Constructing Social Research. The Unity and Diversity of Method. Thousand Oaks: Pine Forge Press.

Ragin, C.C., 2000: Fuzzy-Set Social Science. Chicago: University of Chicago Press.

Ragin, C.C., 2004: Turning the Tables: How Case-Oriented Research Challenges Variable-Oriented Research. S. 123-138 in: H. E. Brady \& D.Collier (Hrsg.), Rethinking Social Inquiry. Diverse Tools, Shared Standards. Lanham et al.: Rowman \& Littlefield.

Ragin, C.C., 2006: Set Relations in Social Research: Evaluating Their Consistency and Coverage. Political Analysis 14: 291-310.

Ragin, C.C., 2008: Redesigning Social Inquiry. Fuzzy Sets and Beyond. Chicago: University of Chicago Press.

Ragin, C.C., 2009: Qualitative Comparative Analysis using Fuzzy Sets (fsQCA). S. 87-121 in: B. Rihoux \& C.C. Ragin (Hrsg.), Configurational Comparative Methods. Qualitative Comparative Analysis (QCA) and Related Techniques. Thousand Oaks und London: Sage.

Ragin, C.C., 2014: Comment: Lucas and Szatrowski in Critical Perspective. Sociological Methodology 44: 80-94.

Ragin, C.C. \& P.C. Fiss, 2009: Net Effects Analysis Versus Configurational Analysis: An Empirical Demonstration. S. 190-212 in: C.C. Ragin (Hrsg.), Redesigning Social Inquiry: Set Relations in Social Research. Chicago: University of Chicago Press.

Ragin, C.C. \& S.I. Strand, 2008: Using Qualitative Comparative Analysis to Study Causal Order. Sociological Methods \& Research 36: 431-441.

Rihoux, B., P. Álamos-Concha, D. Bol, A. Marx \& I. Rezsöhazy, 2013: From Niche to Mainstream Method? A Comprehensive Mapping of QCA Applications in Journal Articles from 1984 to 2011. Political Research Quarterly 66: 175-185.

Rihoux, B. \& C.C. Ragin (Hrsg.), 2009: Configurational Comparative Methods. Qualitative Comparative Analysis (QCA) and Related Techniques. Thousand Oaks und London: Sage.

Roberts, R., R. Gott \& J. Glaesser, 2010: Students' Approaches to Open-Ended Science Investigation: The Importance of Substantive and Procedural Understanding. Research Papers in Education 25: 377-407.

Rohlfing, I., 2012: Case Studies and Causal Inference. An Integrative Framework. Houndmills, Basingstoke: Palgrave Macmillan.

Rohlfing, I., 2015: Why Simulations Are Appropriate for Evaluating Qualitative Comparative Analysis. Quality \& Quantity, online first.

Rohlfing, I. \& C.Q. Schneider, 2013: Formalized Case -Selection for Process Tracing after QCA: The Case of Necessity. Political Research Quarterly 66: 220-235.

Savage, S.V. \& R. Seebruck, 2015: The Road to the Top: A Qualitative Comparative Analysis of Mobility in the 
Elite Labor Market of College Basketball Coaching. Sociological Focus 48: 208-227.

Schneider, C.Q. \& K. Makszin, 2014a: Forms of Welfare Capitalism and Education-Based Participatory Inequality. Socio-Economic Review 12: 437-462.

Schneider, C.Q. \& K. Makszin, 2014b: Online Appendix to Forms of Welfare Capitalism and Education-Based Participatory Inequality. Online verfügbar unter http:// ser.oxfordjournals.org/content/suppl/2014/03/25/mwu 010.DC1

Schneider, C.Q. \& I. Rohlfing, 2013: Combining QCA and Process Tracing in Set-Theoretic Multi-Method Research. Sociological Methods \& Research 42: 559_ 597.

Schneider, C.Q. \& I. Rohlfing, 2014: Clarifying Misunderstandings, Moving Forward: Towards Standards and Tools in Set-Theoretic Methods. Qualitative and Multi-Method Research. Newsletter of the APSA Section for Qualitative and Multi-Method Research 12: 27-34.

Schneider, C.Q. and C. Wagemann, 2012: Set-Theoretic Methods for the Social Sciences. A Guide to Qualitative Comparative Analysis. Cambridge: Cambridge University Press.

Schneider, C.Q. \& C. Wagemann, 2010: Standards of Good Practice in Qualitative Comparative Analysis (QCA) and Fuzzy Sets. Comparative Sociology 9: 397-418.

Seawright, J., 2014: Comment: Limited Diversity and the Unreliability of QCA. Sociological Methodology 44: 118-121.

Shanahan, M.J., S. Vaisey, L.D. Erickson \& A. Smolen, 2008: Environmental Contingencies and Genetic Propensities: Social Capital, Educational Continuation, and Dopamine Receptor Gene DRD2. American Journal of Sociology 114: S260-S286.

Shanahan, M.J., L.D. Erickson, S. Vaisey \& A. Smolen, 2007: Helping Relationships and Genetic Propensities: A Combinatoric Study of DRD2, Mentoring, and Educational Continuation. Twin Research and Human Genetics 10: 285-298.

Skaaning, S.-E., 2011: Assessing the Robustness of CrispSet and Fuzzy-Set QCA Results. Sociological Methods \& Research 40: 391-408.

Stevenson, I., 2013: Does Technology have an Impact on Learning? A Fuzzy Set Analysis of Historical Data on the Role of Digital Repertoires in Shaping the Outcomes of Classroom Pedagogy. Computers \& Education 69: 148-158.
Svevo-Cianci, K.A., S.N. Hart \& C. Rubinson, 2010: Protecting Children from Violence and Maltreatment: A Qualitative Comparative Analysis Assessing the Implementation of U.N. CRC Article 19. Child Abuse \& Neglect 34: 45-56.

Thiem, A., 2014a: Navigating the Complexities of Qualitative Comparative Analysis: Case Numbers, Necessity Relations, and Model Ambiguities. Evaluation Review 38: 487-513.

Thiem, A., 2014b: Mill's Methods, Induction and Case Sensitivity in Qualitative Comparative Analysis: A Comment on Hug. Qualitative and Multi-Method Research. Newsletter of the APSA Section for Qualitative and Multi-Method Research 12: 19-24.

Vaisey, S., 2014: Comment: QCA Works - When Used with Care. Sociological Methodology 44: 108-112.

Vaisey, S., 2009: QCA 3.0: The „Ragin Revolution“ Continues. Contemporary Sociology. A Journal of Reviews 38: 308-312.

Vaisey, S., 2007: Structure, Culture, and Community: The Search for Belonging in 50 Urban Communes. American Sociological Review 72: 851-873.

Verkuilen, J., 2005: Assigning Membership in a Fuzzy Set Analysis. Sociological Methods \& Research 33: 462496.

Wagemann, C., 2015: Qualitative Comparative Analysis. S. 429-453 in: G. Wenzelburger \& R. Zohlnhöfer (Hrsg.), Handbuch Policy-Forschung. Wiesbaden: VS Springer.

Wagemann, C. \& C.Q. Schneider, 2015: Transparency Standards in Qualitative Comparative Analysis. Qualitative and Multi-Method Research. Newsletter of the APSA Section for Qualitative and Multi-Method Research 13: 38-42.

Wagemann, C. \& C.Q. Schneider 2003: Fuzzy Set Qualitative Comparative Analysis (fs/QCA): Ein Zwei-Stufen-Modul. S. 105-134 in: S. Pickel, G. Pickel, H.-J. Lauth und D. Jahn (Hrsg.), Vergleichende politikwissenschaftliche Methoden - Neue Entwicklungen und Diskussionen. Opladen: Westdeutscher Verlag.

Wollebæk, D., 2010: Volatility and Growth in Populations of Rural Associations. Rural Sociology 75: 144166.

Wright, R.A. \& H. Schaffer Boudet, 2012: To Act or Not to Act: Context, Capability, and Community Response to Environmental Risk. American Journal of Sociology 118: 728-777.

\section{Autorenvorstellung}

Jonas Buche, geb. 1982 in Rostock. Studium der Politikwissenschaft, Philosophie und neuesten Geschichte in Kiel und Göteborg (S). Von 2009-2012 Forschungsreferent am Deutschen Forschungsinstitut für öffentliche Verwaltung in Speyer, seit 2012 wissenschaftlicher Mitarbeiter an der Professur für Gesellschaftswissenschaften mit dem Schwerpunkt qualitative empirische Sozialforschung an der Goethe-Universität Frankfurt.

Wichtigste Publikationen: Fuzzy Logic or Fuzzy Application? A Response to Stockemer's Fuzzy Set or Fuzzy Logic? (mit A. Buche \& M.B. Siewert), European Political Science, im Erscheinen; QCA and Business Research: Work in Progress or a Consolidated Agenda? (mit C. Wagemann \& M.B. Siewert), Journal of Business Research, online first. 
Markus B. Siewert, geb. 1980 in Freiburg im Breisgau. Studium der Politikwissenschaft und neueren und neuesten Geschichte in Freiburg. Seit 2012 wissenschaftlicher Mitarbeiter an der Professur für Gesellschaftswissenschaften mit dem Schwerpunkt qualitative empirische Sozialforschung an der Goethe-Universität Frankfurt.

Wichtigste Publikationen: Fuzzy Logic or Fuzzy Application? A Response to Stockemer's Fuzzy Set or Fuzzy Logic? (mit A. Buche \& J. Buche), European Political Science, im Erscheinen; QCA and Business Research: Work in Progress or a Consolidated Agenda? (mit C. Wagemann \& J. Buche), Journal of Business Research, online first; Handbuch Politik USA (hrsg. mit Ch. Lammert \& B. Vormann), Wiesbaden 2015. 